\title{
TOPOLOGIES ON SPACES OF SUBSETS
}

\author{
BY \\ ERNEST MICHAEL
}

1. Introduction. In the first part of this paper ( $\$ \S 2-5)$, we shall study various topologies on the collection of nonempty closed subsets of a topological space ${ }^{(1)}$. In the second part ( $\left.\$ \S 6-8\right)$, we shall apply these topologies to such topics as multi-valued functions and the linear ordering of a topological space.

To facilitate our discussion, we begin by listing some of our principal conventions and notations:

Convention 1.1. Let $X$ be the "base" space. Then

1.1.1. An element of $X$ will be denoted by lower case italic letters (for example, $x$ ).

1.1.2. A subclass of $X$ will be called a set, and will be denoted by upper case italic letters (for example, $E$ ).

1.1.3. A class of sets will be called a collection, and will be denoted by light-face German letters (for example, $\mathfrak{B}$ ).

1.1.4. A class of collections will be called a family, and will be denoted by bold face German letters (for example, $\mathfrak{A}$ ).

Convention 1.2. By a neighborhood of a class, we shall always mean a neighborhood of this class considered as an element of a topological space, not as a subclass of such a space.

Notation 1.3.

1.3.1. A topological space $X$, with topology $T$, will be denoted by $(X, T)$.

1.3.2. A uniform space $X$, with uniform structure $U$, will be denoted by $[X, U]$; the topology which $U$ induces on $X$ will be denoted by $|U|$.

Notation 1.4. Let $(X, T)$ be a topological space. Then

$A(X)=\{E \subset X \mid E$ is not empty $\}$,

$2^{X}=\{E \subset X \mid E$ is closed and not empty $\}$,

$\mathcal{F}_{n}(X)=\left\{E \in 2^{X} \mid E\right.$ has at most $n$ elements $\}$,

$\mathcal{F}(X)=\left\{E \in 2^{x} \mid E\right.$ is finite $\}$,

$\mathcal{C}(X)=\left\{E \in 2^{x} \mid E\right.$ is compact $\}\left({ }^{2}\right)$.

The following notation will be useful for defining and discussing our topologies:

Notation 1.5. If $\left\{U_{\imath}\right\}_{\imath} \in \mathrm{I}$ is a collection of subsets of a topological space $X$,

Presented to the Society, April 29, 1950; received by the editors April 26, 1950 and, in revised form, December 18, 1950.

(1) Such collections are of ten called hyperspaces.

(2) Strictly speaking, we should write $\mathcal{C}(X, T)$ and so forth, since $\mathcal{C}(X)$ depends on $T$. Where no confusion can occur, we shall, however, adopt the simplified notation. 
then $\left\langle U_{\iota}\right\rangle_{\iota} \in \mathrm{I}$ (or $\left\langle U_{1}, \cdots, U_{n}\right\rangle$ if $\mathrm{I}=\{1, \cdots, n\}$ ) denotes $\left\{E \in 2^{x} \mid E\right.$ $\subset U_{\imath} \in \mathrm{I} U_{\iota}$, and $E \cap U_{\imath} \neq \varnothing\left({ }^{3}\right)$ for all $\left.\iota \in \mathrm{I}\right\}$.

The first step towards topologizing a collection of subsets of a topological space $X$ was taken by Hausdorff $[10, \S 28]\left({ }^{4}\right)$, who defined a metric on $2^{x}$ in the case where $X$ is a bounded metric space( $\left.{ }^{5}\right)$. If we extend the Hausdorff metric to $A(X)$, then sets with the same closure are at zero distance from each other; hence the extended distance function is not a metric. When topologizing the subsets of a more general topological space, the situation is quite similar: On $2^{x}$, these topologies have decent separation properties, but when extended to $A(X)$, they fail to be $T_{1}$ (in fact, for all $E \subset X$, any neighborhood of $\bar{E}$ contains $E$ ). We shall therefore, except in $\S 5$, restrict our considerations to $2^{x}$.

One of the features of the Hausdorff metric is that the function( $\left.{ }^{6}\right) i: X$ $\rightarrow 2^{x}$, which maps $x$ into $\{x\}\left(^{3}\right)$, is an isometry. In order to retain analogous properties for more general $X$, it is necessary, first of all, that the one-point subsets of $X$ be elements of $2^{X}$; we shall therefore assume throughout this paper (except in 4.9.1. and 4.9.2) that the base space $X$ is $T_{1}$. We now call a (topology/uniform structure/metric) on $2^{X}$ admissible with respect to a (topology/uniform structure/metric) on $X$ if the function $i$ is a (homeomorphism/uniform isomorphism/isometry). It is reasonable to require that all structures on $2^{x}$ be admissible, and the ones that we are about to define do, in fact, satisfy this requirement.

Guided by the above preliminary observations, we are now ready to topologize $2^{X}$ when $X$ is not a bounded metric space. For uniform spaces, we follow Bourbaki $[1$, p. 96, Ex. 7] in making the following definition.

Definition 1.6. Let $[X, U]$ be a uniform space with index set $A$. For each $E \in 2^{x}$, let $\mathfrak{B}_{\alpha}(E)=\left\langle V_{\alpha}(x)\right\rangle_{x \in E}$. We then define the uniform structure $2^{U}$ on $2^{X}$ to be the one generated by $A$ and the collection $\mathfrak{B}_{\alpha}(E)$, and we call $\left|2^{U}\right|$ the uniform topology. The verification that this actually generates a $T_{1}$ uniform structure on $2^{x}$ is tedious, but entirely straightforward; it is given as an exercise in [1, p. 97, Ex. 7], and will be omitted here. For an arbitrary topological space, we follow Vietoris $[14$, p. 259, $\Re$-Umgebungen] in defining a topology on $2^{X}$ as follows:

Definition 1.7. If $(X, T)$ is a topological space, then the finite topology $2^{T}$ on $2^{X}$ is the one generated by open collections of the form $\left\langle U_{1}, \cdots, U_{n}\right\rangle$ with $U_{1}, \cdots, U_{n}$ open subsets of $X$.

(3) We use $\varnothing$ to denote the null set. We also use $\bar{E}$ (or $\mathrm{Cl}(E)$ if the expression for $E$ is long) to denote the closure of a set $E$, and $E^{\prime}$ (or $X-E$ ) to denote the complement of $E$ (in $X$ ). If $x$ is an element of $X$, we use $\{x\}$ to denote the subset of $X$ whose only element is $x$. Similarly for subclasses or elements of hyperspaces.

(4) Numbers in brackets refer to the bibliography at the end of the paper.

(b) For an extensive study of this metric topology on $2^{X}$ for metric compact $X$, see Kelley [11].

(6) The terms function, map, and mapping are used synonymously in this paper, and do not imply continuity. 
It is easy to see that if $X$ is a bounded metric space, then $2^{U}$ agrees with the Hausdorff metric. The finite topology $2^{T}$, on the other hand, agrees with this metric only if $X$ is compact (see Proposition 3.5); this is no calamity, however, for in some important respects the finite topology behaves much better than the metric topology (see 2.4.1 and 4.10).

$\$ 2$ deals with fundamental properties of the topologies on $2^{x}$. What is probably the most interesting result of this section (Theorem 2.5) can be paraphrased as follows: A compact union of closed sets is closed, and a compact union of compact sets is compact.

$\$ 3$ deals with relationships between the various topologies on $2^{x}$. The most important results are that, for a uniform space $[X, U]$, the uniform and finite topologies agree on $\mathcal{C}(X)$ (Theorem 3.3), and that, for a normal $X$ and a special $U$, they even agree on $2^{X}$ (Theorem 3.4).

In $\S 4$, we investigate what properties of $X$ are carried over to $2^{X}$ or to $\mathcal{C}(X)$. Among the former are compactness (Theorem 4.2), connectedness (Theorem 4.10), and most separation properties in somewhat weakened form (Theorems 4.9.1-4.9.7). As for $\mathcal{C}(X)$, it appears to inherit intact virtually every property of $X$. In addition to the properties just mentioned for $2^{X}$, $\mathcal{C}(X)$ inherits the separation properties in undiluted form (Theorems 4.9.84.9.13), local compactness (Proposition 4.4), and local connectedness (Theorem 4.12).

In $\S 5$, we begin by extending our topologies to $A(X)$ (Definition 5.1), and showing that the function ${ }^{(6)}$ which maps a subset of $X$ into its closure is a retraction (Theorem 5.3). Next we define certain functions among the hyperspaces (Definition 5.5), and derive their properties (Corollary 5.6-Corollary 5.8). Typical among these is that the function $\sigma: \mathcal{A}(\mathcal{A}(X)) \rightarrow \mathcal{A}(X)$, which maps a collection of sets into its union, is continuous (Theorems 5.7.1 and 5.7.2). Next we study the relationships between a function $f: X \rightarrow Y$ and the function it induces among the hyperspaces (Theorem 5.10). We conclude this section by defining a new concept, the saturate of a collection (Definition 5.13), and deriving its properties. An interesting result is that the union of a closed and saturated collection of closed sets is closed (Corollary 5.16).

Before continuing this summary, we define a concept which plays a crucial role in the investigations of the remaining sections.

Definition 1.8. Let $\subseteq \in 2^{X}$. A function $f: \subseteq \rightarrow X$ is called a selection if it is continuous, and if $f(E) \in E$ for all $E \in \subseteq$.

In $\S 6$ we discuss the following question: Given a function $g: Y \rightarrow 2^{X}$, when is it possible to find a continuous $g^{\prime}: Y \rightarrow X$ such that $g^{\prime}(y) \in g(y)$ for all $y \in Y$ ? We attack this problem by dividing it into two parts; the first part deals with the continuity of $g$, while the second deals with the properties of $X$ alone. Among the new questions thus raised, we have the following:

Question 1. For what $X$ does there exist a selection from $2^{X}$ (or from $\mathcal{C}(X)$ ) to $X$ ? 
Question 2. For what $X$ does there exist, for every disjoint covering $\mathfrak{D}$ of $X$ by closed (compact) sets, a selection from $\mathfrak{D}$ to $X$ ?

In $\S 7$ we study Question 1 and related topics. Question 1 itself is completely answered by the following theorem.

ThEOREM 1.9. Among the following statements, 1.9.2 $\rightarrow$ 1.9.1 and 1.9.4 $\rightarrow$ 1.9.3 for any Hausdorff space $(X, T)$. If, moreover, every connected component of $X$ is open, then conversely, 1.9.1 $\rightarrow$ 1.9.2 and 1.9.3 $\rightarrow$ 1.9.4:

1.9.1. There exists a selection $f: 2^{X} \rightarrow X$.

1.9.2. There exists a linear ordering on $X$ such that the order topology is coarser than $T$, and such that every closed $(T)$ set has a first element.

1.9.3. There exists a selection $f: \mathcal{C}(X) \rightarrow X$.

1.9.4. There exists a linear ordering on $X$ such that the order topology is coarser than $T$.

We note that if, in particular, $X$ is a connected Hausdorff space, then 1.9.1 $\rightleftarrows 1$ 1.9.2 and 1.9.3 $\rightleftarrows 1$ 1.9.4. These equivalences should be compared with [ 6 , Theorem 1], where another necessary and sufficient condition for 1.9.4 is given. The chapter concludes with some examples of spaces which do, or do not, satisfy 1.9 .2 or 1.9 .4 .

In $\$ 8$ we begin by showing that certain selection properties are retained under mappings $\left({ }^{6}\right)$ which are continuous, open, and closed. We conclude the section by giving some partial answers to Question 2 .

In the appendix, we begin by re-examining the foundations of the finite topology. This leads us to some new theorems, which in turn lead to new applications. We conclude by outlining a similar program for the uniform case.

2. Fundamental properties of the topologies on $2^{x}$. We begin this section by observing that the topologies $2^{T}$ and $\left|2^{U}\right|$, the uniform structure $2^{U}$, and the Hausdorff metric are all admissible.

We now examine the finite topology.

Proposition 2.1(7). The collections of the form $\left\langle U_{1}, \cdots, U_{n}\right\rangle$, with $U_{1}, \cdots, U_{n}$ open in $X$, form a basis for the finite topology on $2^{X}$.

Proof. (a) $2^{X}=\langle X\rangle$.

(b) Let $\mathfrak{U}=\left\langle U_{1}, \cdots, U_{n}\right\rangle, \mathfrak{B}=\left\langle V_{1}, \cdots, V_{m}\right\rangle$, and let $U=\bigcup_{i=1}^{n} U_{i}$, $V=\bigcup_{i=1}^{m} V_{i}$. Then $\mathfrak{U} \cap \mathfrak{B}=\left\langle U_{1} \cap V, \cdots, U_{n} \cap V, V_{1} \cap U, \cdots, V_{m} \cap U\right)$.Q.E.D.

Our definition of $2^{T}$ is essentially the one given by Vietoris and Choquet. It is easy to verify that it is equivalent to Frink's neighborhood topology (see $[7, \S 12]$ ). Before leaving this topic, we give another definition of $2^{T}$, similar to Frink's, which makes this topology appear especially "natural."

DEFINITION 1.6a. If $(X, T)$ is a topological space, we say that a topology on

$\left(^{7}\right)$ This assertion remains true if $2^{x}$ is replaced by $A(X)$ (and \langle\rangle , if occurring, by \langle\rangle$^{+}$). For the topology on $A(X)$, see Definition 5.1. 
$2^{X}$ is acceptable if in it $\left\{E \in 2^{x} \mid E \subset A\right\}$ is closed for all closed $A \subset X$, and is open for all open $A \subset X$. Then the finite topology $2^{T}$ is the coarsest acceptable topology on $2^{x}$.

Note. The uniform topology is, in general, not acceptable. It might be interesting to study the properties of an arbitrary acceptable topology, but this will not be done in this paper.

The following lemmas deal with simple properties of the finite and uniform topologies:

LemMA $2.2^{(7)}$. Let $(X, T)$ be a topological space. Then:

2.2.1. $\left\{E \in 2^{X} \mid E \subset A\right\}$ is closed in $\left(2^{X}, 2^{T}\right)$ if $A \subset X$ is closed.

2.2.2. $\left\{E \in 2^{X} \mid E \cap A \neq \varnothing\right\}$ is closed in $\left(2^{X}, 2^{T}\right)$ if $A \subset X$ is closed. If $[X, U]$ is a uniform space, then

2.2.1'. $\left\{E \in 2^{X} \mid E \subset A\right\}$ is closed in $\left(2^{X},\left|2^{U}\right|\right)$ if $A \subset X$ is closed.

2.2.2'. $\left\{E \in 2^{X} \mid E \cap A \neq \varnothing\right\}$ is closed in $\left(2^{X},\left|2^{U}\right|\right)$ if $A \subset X$ is closed, provided $A$ is compact.

Proof. The first three statements follow directly from the definition. The last statement follows from the fact that if $A$ and $B$ are closed subsets of a uniform space, one of which is compact, then there exists an index $\alpha$ such that $V_{\alpha}(A) \cap V_{\alpha}(B)=\varnothing$ (see Notation 3.1.2). Q.E.D.

The following assertions follow immediately from the definitions, and are stated without proof:

LEMMA $2.3\left({ }^{7}\right)$.

2.3.1. $\left(\left\langle U_{1}, \cdots, U_{n}\right\rangle \subset\left\langle V_{1}, \cdots, V_{m}\right\rangle\right) \rightleftarrows\left(\bigcup_{i=1}^{n} U_{i} \subset \cup_{i=1}^{m} V_{i}\right.$, and for every $V_{i}$ there exists a $U_{j}$ such that $\left.U_{j} \subset V_{i}\right)$.

2.3.2. In the finite topology on $2^{X}, \mathrm{Cl}\left(\left\langle U_{1}, \cdots, U_{n}\right\rangle\right)=\left\langle\bar{U}_{1}, \cdots, \bar{U}_{n}\right\rangle\left(^{3}\right)$.

2.3.3. If $\left\{U_{\alpha}\right\}_{\alpha \in \mathrm{A}}$ is a basis for the neighborhoods of $x$ in $X$, then $\left\{\left\langle U_{\alpha}\right\rangle\right\}_{\alpha \in \mathrm{A}}$ is a basis for the neighborhoods of $\{x\}$ in $2^{x}$ in either the uniform or the finite topology.

Proposition 2.4( $\left.{ }^{7}\right)$. If $(X, T)$ is a topological space, then:

2.4.1. $\mathcal{F}(X)$ is dense in $\left(2^{X}, 2^{T}\right)$.

2.4.2. If $X$ is Hausdorff, then $\mathcal{F}_{n}(X)$ is closed in $\left(2^{X}, 2^{T}\right)$ for all $n \geqq 1$.

2.4.3. The natural map pr: $X^{n} \rightarrow \mathcal{F}_{n}(X)$, defined by $\mathrm{pr}\left(\left(x_{1}, \cdots, x_{n}\right)\right)$ $=\left\{x_{1}, \cdots, x_{n}\right\}$, is continuous.

Note. (a) If $[X, U]$ is a uniform space, then 2.4.2 and 2.4.3 also hold with the uniform topology on $2^{x}$ (the mapping pr is now, in fact, uniformly continuous). Assertion 2.4.1 is, however, false in this case.

(b) Assertion 2.4.3 is false for infinite products.

(c) If $X$ is not Hausdorff and if $\mathcal{F}_{n}(X) \neq 2^{X}$, then $\mathcal{F}_{n}(X)$ is not dense in $\left(2^{x}, 2^{T}\right)$.

The following theorem generalizes the elementary fact that a finite union of closed (compact) sets is closed (compact). 
THEOREM $2.5\left(^{8}\right)$.

2.5.1. If $(X, T)$ is a regular space, then

$$
\mathfrak{B} \in \mathcal{C}\left(2^{x}, 2^{T}\right) \rightarrow\left(\bigcup_{E} \in \mathfrak{B}\right) \in 2^{x} .
$$

2.5.2. If $(X, T)$ is a topological space, then

$$
\mathfrak{B} \in \mathcal{C}\left(\mathcal{C}(X), 2^{T}\right) \rightarrow\left(\bigcup_{E \in \mathfrak{B}} E\right) \in \mathcal{C}(X) .
$$

2.5.1'. If $[X, U]$ is a uniform space, then

$$
\mathfrak{B} \in \mathcal{C}\left(2^{x},\left|2^{U}\right|\right) \rightarrow\left(\bigcup_{E \in \mathfrak{B}} E\right) \in 2^{x} .
$$

2.5.2'. If $[X, U]$ is a uniform space, then

$$
\mathfrak{B} \in \mathcal{C}\left(\mathcal{C}(X),\left|2^{U}\right|\right) \rightarrow\left(\bigcup_{E \in \mathfrak{B}} E\right) \in \mathcal{C}(X) .
$$

Proof. 2.5.1. Suppose $\mathfrak{B} \in C\left(2^{X}\right)$. Let $A=\cup_{E \in \mathfrak{B}} E$, and let $x \in \bar{A}$. Then for each closed neighborhood $N$ of $X, \mathfrak{B} \cap\left\{E \in 2^{x} \mid E \cap N \neq \varnothing\right\}$ is a closed subcollection of $\mathfrak{B}$ (see 2.2.2), and the family of all such subcollections has the finite intersection property. Since $\mathfrak{B}$ is compact, this family has a nonempty intersection $\mathfrak{D C B}$. But since $X$ is regular, $x$ is an element of every element of $\mathfrak{D}$, whence $x \in A$.

2.5.2. Suppose $A=\bigcup_{E \in \mathfrak{B}} E$, with $\mathfrak{B} \subset \mathcal{C}(\mathcal{C}(X))$. Let $\mathfrak{u}$ be a collection of open subsets of $X$ which covers $A$. Now let $E \in \mathfrak{B}$; then $E$ is a compact subset of $X$, and hence there exists a finite subcollection $\left\{U_{E, 1}, \cdots, U_{E, n(E)}\right\}$ of $\mathfrak{U}$ which covers $E$, and all of whose elements intersect $E$. Hence, for each $E \in \mathfrak{B}, \mathfrak{U}_{E}=\left\langle U_{E, 1}, \cdots, U_{E, n(E)}\right\rangle$ is an open neighborhood of $E$, and therefore $\left\{\mathfrak{U}_{E}\right\}_{E \in \mathfrak{B}}$ is a covering of $\mathfrak{B}$ by open collections. But $\mathfrak{B}$ is compact, so there exists a finite subcollection $\left\{E_{1}, \cdots, E_{m}\right\}$ of $\mathfrak{B}$ such that $\left\{\mathfrak{U}_{E_{1}}, \cdots, \mathfrak{U}_{E_{m}}\right\}$ is a covering of $\mathfrak{B}$. Hence, finally, $\left\{\mathfrak{U}_{E_{i}, j}\right\}_{i=1, \ldots, m}^{j=1, \ldots, n\left(E_{i}\right)}$ is a finite subcollection of $\mathfrak{U}$ which covers $A$.

2.5.1'. Suppose that $\mathfrak{B} \in \mathcal{C}\left(2^{x}\right)$. Let $A=\cup_{E \in \mathfrak{B}} E$, and let $x \in A^{\prime}$. For each $E \in \mathfrak{B}$, pick an index $\alpha(E)$ such that $x \notin V_{\alpha(E)}(E)$; then pick a $\beta(E) \in \Sigma(\alpha(E))$ and a $\gamma(E) \in \Sigma\left(\beta(E)\right.$ ) (see Notation 3.1). Then $\left\{\mathfrak{B}_{\gamma(E)}(E)\right\}_{E \in \mathfrak{B}}$ is a covering of $\mathscr{B}$ by open collections, and hence there exists a finite subcovering $\left\{\mathfrak{B}_{\gamma\left(E_{i}\right)}\left(E_{i}\right)\right\}_{i=1}^{n}$. Let $\gamma=\max \left\{\gamma\left(E_{i}\right)\right\}$. It follows easily that $x \notin V_{\gamma}(A)$, and hence that $x$ has a neighborhood which is disjoint from $A$.

2.5.2'. This follows directly from 2.5.2 and Theorem 3.3 of the next section. Q.E.D.

(8) For a stronger version of this assertion, see the Appendix. 
Theorem 2.5 and its extension in the appendix have many applications, where they reveal the basic similarity between some apparently unrelated problems. In particular, Theorem 2.5 yields especially simple and transparent proofs for the following theorems in group theory:

(1) If $G$ is a topological group, $A$ a compact subset of $G$, and $B$ a compact (closed) subset of $G$, then $A B$ is compact (closed).

(2) If $G$ is a topological group, $H$ a compact subgroup of $G, \phi$ the canonical mapping of $G$ onto $G / H$, and (E) a compact subcollection of $G / H$, then $\phi^{-1}(\mathbb{E})$ is compact in $G$.

To prove the first theorem, let $U$ be the right uniform structure on $G$. Then the function $f:[G, U] \rightarrow\left[2^{G}, 2^{U}\right]$, defined by $f(x)=x B$, is clearly uniformly continuous. Hence $f(A)$ is compact; since $A B=\mathrm{U}_{E \in f(G)} E$, the conclusion follows from 2.5.2' $\left(2.5 .1^{\prime}\right)$. To prove the second theorem, we observe that $\phi^{-1}(\mathfrak{})=\bigcup_{E \in \mathbb{E}} E$; the conclusion now follows from 2.5.2' and the first part of the following proposition.

Proposition 2.6. Let $G$ be a topological group, $H$ a closed, normal subgroup of $G$. If $U$ is the right (left) uniform structure on $G$, then the right (left) uniform structure on $G / H$ is the one induced on $G / H$ as a subcollection of $\left[2^{G}, 2^{U}\right]$. Furthermore, $G / H$ is a closed subcollection of $\left(2^{G},\left|2^{U}\right|\right)$ for either $U$.

Proof. The first assertion is [2, p. 31, Ex. 5]. Its verification, as well as that of the second assertion, is straightforward, and we omit it. Q.E.D.

Proposition 2.6 leads us to consider the factor topology (topologie quotient in $[1$, p. 52, Definition 1]). In general, the following proposition, whose proof we omit, is all we can assert; in special cases we have better results, such as 2.6 above, 5.10.4, and 5.11.1.

Proposition 2.7( $\left.{ }^{8}\right)$. If $\mathfrak{D}$ is a disjoint covering of a topological space $(X, T)$ by closed subsets, then the factor topology on $\mathfrak{D}$ is coarser than the relative topology induced on $\mathfrak{D}$ by $2^{T}$.

The following proposition is analogous to Theorem 2.5. The proof is elementary, and we omit it.

Proposition $2.8\left(^{7}\right)\left({ }^{8}\right)$. If $\mathfrak{B}$ is a collection of subsets of $X$ which is (disjoint/a subcollection of $2^{X}$ ) and connected in the (factor/finite) topology, and (all/one) of whose elements are connected, then $\mathrm{U}_{E \in \mathfrak{B}} E$ is connected.

We conclude this section with some examples. In all cases, $U$ will denote the natural uniform structure on $X$.

Example 2.9. Let $X$ be the plane, and let $\mathfrak{D}$ be the disjoint covering of $X$ whose elements are the horizontal lines in the open upper half-plane, and the vertical half-lines in the closed lower half-plane. Then $2^{|v|}$ is discrete on $\mathfrak{D}$, $\left|2^{U}\right|$ is coarser on $\mathfrak{D}$ but still disconnected, while the factor topology on $\mathfrak{D}$ is coarser yet and is connected. 
Example 2.10. Let $A$ be the graph of $f(x)=1 / x, Y$ the $y$-axis, $X=A \cup Y$, and $\mathfrak{D}$ the covering of $X$ whose elements are $A$ and the sets $\{x\}$ with $z \in Y$. Then $2^{|U|}$ and the factor topology agree on $\mathfrak{D}$, and are coarser there than $\left|2^{U}\right| ; A$ is an isolated element of $\mathfrak{D}$ in the former topologies, but not in the latter.

Example 2.11. Let $X$ be the real line, $\mathfrak{B}$ the collection of all closed (finite or infinite) intervals. Then $X$ is an isolated element of $\left(\mathfrak{B},\left|2^{U}\right|\right)$, but not of $\left(\mathfrak{B}, 2^{|U|}\right)$.

Example 2.12. Let $X$ be the real line, $\mathfrak{B}$ the collection of the sets $\{n, 1 / n\}$ $(n=1,2, \cdots)$. Then $2^{|U|}$ and $\left|2^{U}\right|$ agree on $\mathscr{B}$, and $\mathfrak{B}$ is a closed (with either topology on $2^{x}$ ) collection of compact sets whose union is not closed.

It is apparent from the above examples that, except for Proposition 2.7, our various topologies are, in general, incomparable on $2^{x}$ and its subcollections. Nevertheless, some simple and useful relations hold between the finite and uniform topologies, and the next section is devoted to their study.

3. Relations between the various topologies on $2^{x}$. For use in some of the proofs of this section, we introduce the following notation for uniform spaces:

Notation 3.1. Let $[X, U]$ be a uniform space, with index set $\mathrm{A}$. Then, for all $\alpha \in \mathrm{A}$ :

3.1.1. $\Sigma(\alpha)=\left\{\beta \in \mathrm{A} \mid x, y, \quad z \in X: \rightarrow: x \in V_{\beta}(z), \quad y \in V_{\beta}(z) \rightarrow x \in V_{\alpha}(z)\right\}$ (see $[16$, p. 7$])$.

3.1.2. If $E \subset X$, then $V_{\alpha}(E)=\bigcup_{x \in E} V_{\alpha}(x)$.

We now state and prove the fundamental lemma of this section.

Lemma 3.2. Suppose $[X, U]$ is a uniform space, and let $E \in 2^{X}$. Then (see below) $3.2 .1 \rightleftarrows 3.2 .2$, and 3.2.3 $\rightleftarrows 3.2 .4$.

3.2.1. Any $\left|2^{U}\right|$-neighborhood of $E$ contains a $2^{|U|}$-neighborhood of $E$.

3.2.2. $E$ is totally bounded $\left({ }^{9}\right)$.

3.2.3. Any $2^{|U|}$-neighborhood of $E$ contains a $\left|2^{U}\right|$-neighborhood of $E$.

3.2.4. If $F \in 2^{X}$ is disjoint from $E$, then there exists an $\alpha$ such that $V_{\alpha}(E)$ is disjoint from $F$.

Proof. 3.2.1 $\rightarrow$ 3.2.2. Let $\alpha \in \mathrm{A}$, and pick $\beta \in \Sigma(\alpha)$. Then there exists a neighborhood $\left\langle U_{1}, \cdots, U_{n}\right\rangle$ of $E$ which is contained in $\mathfrak{B}_{\beta}(E)$. Let $x_{i} \in U_{i}$ $\cap E(i=1, \cdots, n)$. We assert that the sets $V_{\alpha}\left(x_{1}\right), \cdots, V_{\alpha}\left(x_{n}\right)$ cover $E$. For let $x \in E$. Then for some $i$, say $i=k, U_{i} \subset V_{\beta}(x)$. Hence $x_{k} \in V_{\beta}(x)$, and hence $x \in V_{\alpha}\left(x_{k}\right)$.

3.2.2 $\rightarrow$ 3.2.1. Let $\mathfrak{B}_{\alpha}(E)$ be a $\left|2^{U}\right|$-neighborhood of $E$. Pick $\beta \in \Sigma(\alpha)$, $\gamma \in \Sigma(\beta)$, and $\delta \in \Sigma(\gamma)$. Then there exist points $x_{1}, \cdots, x_{n}$ in $E$ such that the sets $V_{\delta}\left(x_{1}\right), \cdots, V_{\delta}\left(x_{n}\right)$ cover $E$. Hence for each $x \in E$, there exists an $i$, say $i=k$, such that $x \in V_{\delta}\left(x_{i}\right)$. Hence $x_{k} \in V_{\gamma}(x)$, and hence $V_{\delta}\left(x_{k}\right) \subset V_{\alpha}(x)$. There-

(9) A uniform space $X$ is totally bounded if for every index $\alpha$ there exists a finite subset $\left\{x_{1}, \cdots, x_{n}\right\}$ of $X$ such that $X=\cup_{i=1}^{n} V_{\alpha}\left(x_{i}\right)$. 
fore, finally, $\left\langle V_{\delta}\left(x_{1}\right), \cdots, V_{\delta}\left(x_{n}\right)\right\rangle$ is a $2^{|U|}$-neighborhood of $E$ which is contained in $\mathfrak{B}_{\boldsymbol{\alpha}}(E)$.

3.2.3 $\rightarrow$ 3.2.4. Let $F \in 2^{x}$ be disjoint from $E$. Then $\left\langle F^{\prime}\right\rangle$ is a $2^{|U|} \mid$-neighborhood of $E$, and hence there exists an $\alpha \in \mathrm{A}$ such that $\mathfrak{B}_{\alpha}(E) \subset\left\langle F^{\prime}\right\rangle$. But then $V_{\alpha}(E) \subset F^{\prime}$, as asserted.

3.2.4 3.2.3. Let $\left\langle U_{1}, \cdots, U_{n}\right\rangle$ be a $2^{|U|}$-neighborhood of $E$. Let $\alpha \in \mathrm{A}$ be such that $V_{\alpha}(E) \cap\left(\bigcup_{i=1}^{n} U_{i}\right)^{\prime}=\varnothing$. Let $x_{i} \in U_{i} \cap E(i=1, \cdots, n)$, and pick $\beta$ such that $V_{\beta}\left(x_{i}\right) \subset U_{i}(i=1, \cdots, n)$. Let $\gamma \geqq \max (\beta, \alpha)$. Then $\mathfrak{B}_{\gamma}(E)$ $\subset\left\langle U_{1}, \cdots, U_{n}\right\rangle$. Q.E.D.

We now draw several conclusions from Lemma 3.2. The first of these, Theorem 3.3, has the widest application; by virtue of it, any theorem proved about $\mathcal{C}(X)$ using either the finite or the uniform topology will automatically be valid in both topologies. In Theorem 3.4, we consider a special uniform structure on a normal space, and our conclusion is much stronger than that of Theorem 3.3; we use Theorem 3.4 in the proof of Theorem 4.9.5.

THEOREM 3.3. If $[X, U]$ is a uniform space, then $2^{|U|}$ and $\left|2^{U}\right|$ agree on $\mathcal{C}(X)$.

Proof. This follows from Lemma 3.2 and the fact that if $E \subset X$ is compact, then $E$ satisfies conditions 3.2.2 and 3.2.4 [1, p. 111, Proposition 2]. Q.E.D.

THEOREM 3.4. If $X$ is normal, and if $U$ is the uniform structure induced on $X$ by the Stone-Čech compactification, then $2^{|U|}$ and $\left|2^{U}\right|$ agree on $2^{X}$.

Proof. We will prove this theorem by showing that every $E \in 2^{x}$ satisfies conditions 3.2.2 and 3.2.4. Let $\bar{X}$ be the Stone-Cech compactification of $X$. Since $[X, U]$ is precompact, it is totally bounded [1, p. 111, Theorem 4], and so is every $E \in 2^{X}$. Let $E$ and $F$ be disjoint, closed subsets of $X$, and let $\bar{E}$ and $\bar{F}$ be their closures in $\bar{X}$. Then we assert that $\bar{E} \cap \bar{F}=\varnothing:$ For suppose that there exists an $x_{0} \in \bar{E} \cap \bar{F}$. By normality, there exists an $f: X \rightarrow[0,1]$ such that $f(E)=0$ and $f(F)=1$. Now by the Stone-Čech theorem, $f$ can be extended to a continuous function $\bar{f}$ on $\bar{X}$; but no such $\bar{f}$ could be continuous at $x_{0}$. This proves the assertion. Now since $\bar{E}$ and $\bar{F}$ are disjoint compact subsets of a uniform space, there exists an index $\alpha$ such that $\bar{F} \cap V_{\alpha}(\bar{E})=\varnothing[1$, p. 111, Proposition 2]. Hence $F \cap V_{\alpha}(E)=\varnothing$. Q.E.D.

In contrast to Theorem 3.4, we finally have the following proposition.

Proposition 3.5. If the uniform structure of $[X, U]$ is metrizable, then $2^{|U|}$ agrees with $\left|2^{U}\right|$ on $2^{X}$ if and only if $(X,|U|)$ is compact.

Proof. If $X$ is compact, then $2^{|U|}$ agrees with $\left|2^{U}\right|$ on $2^{x}$ by Theorem 3.3. Suppose, conversely, that $2^{|U|}$ and $\left|2^{U}\right|$ agree on $2^{X}$. Since $\left|2^{U}\right|$ is metrizable by 4.1.1, the same is true of $2^{|U|}$, whence $(X,|U|)$ is compact by Theorem 4.6. Q.E.D.

This completes our study of the relations between the finite and the uni- 
form topologies. We conclude this section with the following proposition, which was already announced in the introduction, and which follows from the definitions [3, p. 29, Ex. 7].

Proposition 3.6. Let $X$ be a bounded metric space, and let $U$ be the uniform structure which the metric induces on $X$. Then the uniform structure which the Hausdorff metric induces on $2^{X}$ is identical with $2^{U}$.

4. Relationships between properties of $X$ and $2^{X}$ (or $\mathcal{C}(X)$ ). We begin by collecting some results about uniform spaces.

Proposition 4.1. Let $[X, U]$ be a uniform space.

4.1.1. $[X, U]$ is metrizable $\rightleftarrows\left[2^{X}, 2^{U}\right]$ is metrizable.

4.1.2. $[X, U]$ is totally bounded $\rightleftarrows\left[2^{X}, 2^{U}\right]$ is totally bounded.

4.1.3. Suppose that $[X, U]$ is metrizable. Then $[X, U]$ is complete $\rightleftarrows\left[2^{x}, 2^{U}\right]$ is complete.

4.1.4. There exist (nonmetrizable!) complete $[X, U]$ for which $\left[2^{x}, 2^{U}\right]$ is not complete.

Proof. To prove the nontrivial half of 4.1 .1 , we re-metrize $[X, U]$ by $\rho^{\prime}=\rho /(1+\rho)$, and then apply Proposition 3.6. For the nontrivial parts of 4.1.2 and 4.1.3, see [1, p. 114, Ex. 5], and [12, p. 198] or [3, p. 29, Ex. 7] respectively. Finally, 4.1.4 follows from the last part of Proposition 2.6 and the footnote on p. 37 of [2]. (This proof for 4.1 .4 was suggested to the author by J. Dieudonné.) Q.E.D.

Throughout the remainder of this section, $2^{x}$ will be assumed to carry the finite topology.

The hard part of the following fundamental theorem was first proved by Vietoris [14].

TheOREM 4.2. $X$ is compact $\rightleftarrows 2^{X}$ is compact.

Proof. If $X$ is compact, then $2^{X}$ is proved compact by using Alexander's lemma, which asserts that a space is compact provided every covering by sub-basic open sets has a finite subcovering; see [7, Theorem 15(3)]. Suppose, conversely, that $2^{x}$ is compact, and let $\left\{U_{\alpha}\right\}$ be a covering of $X$ by open sets. Then $\left\{\left\langle X, U_{\alpha}\right\rangle\right\}$ is a covering of $2^{X}$ by open collections, whence there exists a finite subcovering $\left\{\left\langle X, U_{1}\right\rangle, \cdots,\left\langle X, U_{n}\right\rangle\right\}$. But then $\left\{U_{1}, \cdots, U_{n}\right\}$ is a finite subcovering for $X$. Hence $X$ is compact. Q.E.D.

Observe that in the second part of the above proof, we actually proved that if $2^{x} \supset \subseteq \supset \mathcal{F}_{1}(X)$, and if $\subseteq$ is compact, then $X$ is compact. Observe also that this part of the proof is unnecessary if $X$ is assumed to be Hausdorff, since a closed subset of a compact space is compact (see 2.4.2); a similar remark applies to 4.4 .1 below.

Observe that a uniform space $[X, U]$ is compact if and only if $\left[2^{x}, 2^{U}\right]$ is compact. This follows from Theorems 4.2 and 3.3 ; it can also be proved 
directly [1, p. 114, Ex. 6].

The following lemma and its consequences deal with local compactness.

LEMMA 4.3.

4.3.1. If $X$ is locally compact and $A \in \mathcal{C}(X)$, then there exists an open $U \supset A$ such that $\bar{U}$ is compact.

4.3.2. $A \in 2^{X}$ has a compact neighborhood in $2^{X}$ if and only if $A$ is compact.

Proof. 4.3.1. This is trivial.

4.3.2. (a) To prove the "if" assertion, let $U$ be the open set whose existence is asserted in 4.1.1. Then $\langle\bar{U}\rangle=\mathrm{Cl}(\langle U\rangle)$ is a compact neighborhood of $A$ (see Theorem 4.2).

(b) We shall outline the proof of the converse. Let $B \subset X$ be not compact, and let $\mathfrak{U}=\left\langle U_{1}, \cdots, U_{n}\right\rangle$ be a neighborhood of $B$. Let $U=\bigcup_{i=1}^{n} U_{i}$; then $\bar{U}$ is not compact. To complete the proof we must show that, if $\overline{\mathfrak{u}}$ were compact, then $\bar{U}$ would be compact. This demonstration is elementary but dreary, and is omitted. Q.E.D.

Proposition 4.4.

4.4.1. $X$ is locally compact $\rightleftarrows 2^{X}$ is locally compact.

4.4.2. If $X$ is locally compact, then $\mathcal{C}(X)$ is open in $2^{X}$.

Proof. 4.4.1. If $X$ is locally compact, then $2^{x}$ is locally compact by the "if" part of 4.3.2. Suppose, conversely, that $2^{x}$ is locally compact, and let $x \in X$. By Lemma 2.3, there exists a neighborhood $V$ of $x$ such that $\mathrm{Cl}(\langle V\rangle$ $\cap \mathcal{C}(X))$ (closure relative to $\mathcal{C}(X))$ is compact. Then $\mathcal{f}_{1}(\bar{V}) \subset \mathrm{Cl}(\langle V\rangle \cap \mathcal{C}(X))$ $C\left(2^{\bar{v}}, 2^{T} \mid 2^{\bar{v}}\right)=\left(2^{\bar{V}}, 2^{T \mid} \mid \bar{v}\right)$, and hence $\bar{V}$ is compact by the comment following Theorem 4.2.

4.4.2. This follows immediately from 4.3 .1 and 2.2.2. Q.E.D.

We shall study next the question of countability and metrizability. We call a topological space separable if it has a countable dense subset; we call it second (first) countable if there is a countable basis for the open sets (for the neighborhoods of each point).

Proposition 4.5.

4.5.1. $X$ is separable $\rightleftarrows 2^{X}$ is separable.

4.5.2. $X$ is second countable $\rightleftarrows \mathcal{C}(X)$ is second countable.

4.5.3. $X$ is first countable $\rightleftarrows \mathcal{C}(X)$ is first countable.

Proof. The implications pointing to the left are all obvious. Those pointing to the right all follow easily from the fact that the family of finite subclasses of a countable class is countable (and from 2.4.1 in the case of 4.5.1). Q.E.D.

TheOREM $4.6\left({ }^{7}\right)$. If $\left(2^{X}, 2^{T}\right)$ is either metric or second countable, then $(X, T)$ is compact.

Proof. Suppose $X$ were not compact. Then, in either case, it is not count- 
ably compact, and hence there exists a countably infinite $A \subset X$ with no limit points. Hence $A$ is closed in $X, 2^{A} C 2^{X}$, and $A$ is discrete in the relative topology $T \mid A$. Now it is easy to see that $2^{T \mid A}$ is identical with the relative topology $2^{T} \mid 2^{A}$ which $2^{T}$ induces on $2^{A}$; for the rest of the proof, we shall assume that $2^{A}$ carries this topology. Now $2^{A}$ is separable (see 4.5.1), so in either case $2^{A}$ is second countable. We complete the proof by showing that this is impossible. For let $\left\{\mathfrak{u}_{\alpha}\right\}$ be a basis for $2^{A}$, and for each $E \subset A$, pick a basis element $\mathfrak{U}_{E}$ such that $E \in \mathfrak{U}_{E} \subset\langle E\rangle$. Now if $E_{1} \neq E_{2}$, say $E_{2}-E_{1} \neq \varnothing$, then $E_{2} \in \mathfrak{U}_{E_{2}}$ and $E_{2} \notin \mathfrak{U}_{E_{1}}$; hence $\mathfrak{U}_{E_{1}} \neq \mathfrak{U}_{E_{2}}$. It follows that $\left\{U_{\alpha}\right\}$ cannot be countable, for $A$ has more than countably many subsets. Q.E.D.

The next proposition and its corollary deal with the extension of functions from $X$ to $2^{X}$. Proposition 4.7 is used in the proof of 4.9.4 and 4.9.9. We omit the proofs, which consist of straightforward verification.

Proposition 4.7( $\left.{ }^{7}\right)$. Let $X$ be a space, $\bar{R}$ the extended real line (that is, $R$ with $+\infty$ and $-\infty$ added, in the order topology $[2, p .86])$, and $f: X \rightarrow \bar{R}$. Define $f_{+}: 2^{X} \rightarrow \bar{R}$ and $f_{-}: 2^{X} \rightarrow \bar{R}$ by $f_{+}(E)=\sup _{x \in E} f(x), f_{-}(E)=\inf _{x \in E} f(x)$. Then $f_{+}$and $f_{-}$have the same bounds (finite or infinite) as $f$. If $T$ is a topology (resp. $U$ a uniform structure) on $X$, and if $f$ is continuous (resp. has its range in $R$ and is uniformly continuous) with $T$ (resp. $U$ ) on $X$, then $f_{+}$and $f_{-}$are continuous (resp. uniformly continuous, provided their range is contained in $R$ ).

COROLLARY 4.8. Let $(X, T)$ be a topological space ( $[X, U]$ a uniform space), considered as a subspace of $\left(2^{X}, 2^{T}\right)$ (resp. $\left.\left[2^{X}, 2^{U}\right]\right)$. Then any continuous (uniformly continuous) real-valued function $f$ on $X$ can be extended to a continuous (uniformly continuous) real-valued function of $\mathcal{C}(X)$; if $f$ is finitely bounded above or below, then $f$ can be so extended to all of $2^{X}$.

Our next theorem deals with separation properties of $2^{x}$ and $\mathcal{C}(X)$.

THEOREM 4.9.

4.9.1. $2^{X}$ is always $T_{0}$.

4.9.2. $X$ is $T_{1} \rightarrow 2^{X}$ is $T_{1}$. (The converse is false.)

4.9.3. $X$ is regular $\rightleftarrows 2^{X}$ is Hausdorff.

4.9.4. $X$ is completely regular $\rightleftarrows 2^{X}$ is a Stone space $\left({ }^{10}\right)$.

4.9.5. The following are equivalent:

(a) $X$ is normal;

(b) $2^{X}$ is completely regular;

(c) $2^{x}$ is regular.

4.9.6. $X$ is compact Hausdorff $\rightleftarrows 2^{X}$ is compact Hausdorff.

4.9.7. The following are equivalent:

(a) $X$ is compact and metrizable;

(b) $X$ is second countable, compact, and Hausdorff;

${ }^{(10)}$ A topological space $X$ is called a Stone space if, whenever $x_{0}$ and $x_{1}$ are distinct points in $X$, there exists a continuous real-valued function on $X$ such that $f\left(x_{0}\right)=0, f\left(x_{1}\right)=1$. 
(c) $2^{X}$ is compact and metrizable;

(d) $2^{x}$ is second countable, compact, and Hausdorff;

(e) $2^{X}$ is metrizable;

(f) $2^{x}$ is second countable and Hausdorff.

4.9.8. $X$ is Hausdorff $\rightleftarrows \mathcal{C}(X)$ is Hausdorff.

4.9.9. $X$ is a Stone space $\rightleftarrows \mathcal{C}(X)$ is a Stone space $\left({ }^{10}\right)$.

4.9.10. $X$ is regular $\rightleftarrows \mathcal{C}(X)$ is regular.

4.9.11. $X$ is completely regular $\rightleftarrows \mathrm{C}(X)$ is completely regular.

4.9.12. $X$ is compact (locally compact) Hausdorff $\rightleftarrows \mathcal{C}(X)$ is compact (locally compact) Hausdorff.

4.9.13. $X$ is metrizable $\rightleftarrows \mathcal{C}(X)$ is metrizable.

Proof. 4.9.1. If $A, B \in 2^{x}, B-A \neq \varnothing, x \in B-A$, then $B \in\left\langle X, A^{\prime}\right\rangle, A$ $\notin\left\langle X, A^{\prime}\right\rangle$.

4.9.2. Given $A, B \in 2^{x}, B-A \neq \varnothing, x \in B-A$. Then (a) $B \in\left\langle X, A^{\prime}\right\rangle$, $A \notin\left\langle X, A^{\prime}\right\rangle$; (b) $A \in\left\langle\{x\}^{\prime}\right\rangle, B \notin\left\langle\{x\}^{\prime}\right\rangle$.

As a counter example for the converse, consider a many-point space on which the only open sets are the whole space and the null set.

4.9.3. (a) Suppose $X$ is regular. Given $A, B \in 2^{x}, B-A \neq \varnothing, x \in B-A$, let $U, V$ be open sets such that $A \subset U, x \in V$, and $U \cap V=\varnothing$. Then $A \in\langle U\rangle$, $B \in\langle X, V\rangle$, and $\langle U\rangle \cap\langle X, V\rangle=\varnothing$.

(b) Suppose $X$ is not regular. Then there exists an $A \in 2^{X}$, and an $x \notin A$, such that $A$ and $x$ cannot be separated by open sets in $X$. But then it follows easily that $A$ and $A \cup\{x\}$ cannot be separated by open collections in $2^{X}$, whence $2^{X}$ is not Hausdorff.

4.9.4. (a) Suppose $X$ is completely regular. Let $A, B$ be distinct elements of $2^{x}$, and suppose that $B-A \neq 0, x \in B-A$. Let $f$ be a continuous function from $X$ to the interval $[0,1]$ which is 0 at $x$ and 1 on $A$. Then, by Proposition 4.7, the function $f_{+}(E)=\sup _{x \in E} f(x)$ is continuous, and it is clearly 1 at $B$ and 0 at $A$.

(b) Suppose $2^{X}$ is a Stone space. Let $A \subset X$ be closed, and let $x \in X-A$. Let $F$ be a continuous function from $2^{x}$ to the interval $[0,1]$ which is 1 at $A$ and 0 at $A \cup\{x\}$. Let $f: X \rightarrow[0,1]$ be defined by $f(x)=F(A \cup\{x\})$. Then $f$ is clearly continuous, and furthermore $f$ is 1 on $A$ and 0 at $x$.

4.9.5. $\mathrm{a} \rightarrow \mathrm{b}$. This follows from Theorem 3.4 .

$\mathrm{b} \rightarrow \mathrm{c}$. This is obvious.

$\mathrm{c} \rightarrow$ a. Suppose that $2^{x}$ is regular. Let $A \in 2^{x}$, and let $U$ be an open set containing $A$. Now $\langle U\rangle$ is an open neighborhood of $A$ and, therefore, since $2^{x}$ is regular, there exists an open neighborhood $\left\langle V_{1}, \cdots, V_{n}\right\rangle$ of $A$, such that $\left\langle\bar{V}_{1}, \cdots, \bar{V}_{n}\right\rangle \subset\langle U\rangle$ (see 2.3.2). Let $V=\bigcup_{i=1}^{n} V_{i}$. Then $A \subset V$, and $\bar{V} \subset U$ (see 2.3.1).

4.9.6. This follows immediately from 4.2 and 4.9 .3 above.

4.9.7. That $\mathrm{a} \rightleftarrows \mathrm{b}$ and $\mathrm{c} \rightleftarrows \mathrm{d}$ is well known. That $\mathrm{a} \rightarrow \mathrm{c}$ follows from 4.2 , 3.3, and 4.1.1, while $\mathrm{b} \rightarrow \mathrm{d}$ follows from 4.5.2, 4.2, and 4.9.3. That $\mathrm{c} \rightarrow \mathrm{e}$ and 
$\mathrm{d} \rightarrow \mathrm{f}$ is obvious. Finally $\mathrm{e} \rightarrow$ a follows from one half of Theorem 4.6, while $\mathrm{f} \rightarrow \mathrm{b}$ follows from the other half.

In the remaining assertions of this theorem, the implications pointing to the left all follow from the fact that $X$ is homeomorphic to a subcollection of $\mathcal{C}(X)$. It remains to prove the implications pointing to the right.

4.9.8. This goes like 4.9.3 $\rightarrow$, remembering that, in a Hausdorff space, a point and a disjoint compact set can be separated by open sets.

4.9.9. The proof proceeds just like the proof of 4.9.4, if we remember that in a Stone space a point and a disjoint compact set can be separated by a continuous function.

4.9.10. Let $A \in \mathcal{C}(X)$; let $\left\langle U_{1}, \cdots, U_{n}\right\rangle$ be a neighborhood of $A$, and let $U=\bigcup_{i=1}^{n} U_{i}$. It suffices to show that we can find a neighborhood $\left\langle V_{1}, \cdots, V_{n}\right\rangle$ of $A$ whose closure is contained in $\left\langle U_{1}, \cdots, U_{n}\right\rangle$. Now $A \subset U$, and since $A$ is compact and $X$ is regular, there exists an open $V \supset A$ such that $\bar{V} \subset U$. Let $x_{i} \in U_{i} \cap A(i=1, \cdots, n)$. Since $X$ is regular, there exists, for each $i$, a neighborhood $V_{i}$ of $x_{i}$ such that $\bar{V}_{i} \subset U_{i}(i=1, \cdots, n)$. But then $\left\langle V, V_{1}, \cdots, V_{n}\right\rangle$ is a neighborhood of $A$, and (see 2.3.2)

$$
\mathrm{Cl}\left(\left\langle V, V_{1}, \cdots, V_{n}\right\rangle\right)=\left\langle\bar{V}, \bar{V}_{1}, \cdots, \bar{V}_{n}\right\rangle \subset\left\langle U_{1}, \cdots, U_{n}\right\rangle .
$$

4.9.11. Let $U$ be a uniform structure on $X$ such that $|U|=T$. By Theorem $3.3,2^{T}$ is equivalent to $\left|2^{U}\right|$ on $\mathcal{C}(X)$, when $\left(\mathcal{C}(X), 2^{T}\right)$ is completely regular.

4.9.12. If $X$ is compact (locally compact) Hausdorff, then so is $\mathcal{C}(X)$ by 4.9.6 (by 4.4.1 and 4.9.8). If $\mathcal{C}(X)$ is compact (locally compact) Hausdorff, then so is $X$ by 2.4 .2 .

4.9.13. Let $U$ be a metrizable uniform structure on $X$ such that $|U|=T$. Then, again, $2^{T}=\left|2^{U}\right|$ on $\mathcal{C}(X)$. The assertion now follows from 4.1.1. Q.E.D.

Note. 4.9.5 $a \rightarrow c$ could be proved directly without using Theorem 3.4; see the proof of 4.9.10.

The remainder of this section will deal with connectivity. A feature of $2^{x}$ with the Hausdorff metric (and with $X$ compact) is that it has stronger connectivity properties than $X$ itself (see [11], especially the introduction). We shall not go into analogous results in this paper. We shall also not study the properties of the collection of connected, respectively locally connected, subsets of $X$ (see [5], [11], and [15]).

When studying connectivity, it is more convenient to look at all of $A(X)$ than at $2^{x}$. There is an obvious extension of the finite topology to $\mathcal{A}(X)$ (see Definition 5.1 for definition and notation), and for the remainder of this section, we shall assume that $\mathcal{A}(X)$ carries this topology.

THEOREM 4.10. Let $\mathcal{F}(X) \subset \subseteq \subset \mathcal{C} A(X)$. If one of the spaces $X, \mathcal{F}_{n}(X)$ $(n=1,2, \cdots)$, or $\subseteq$ is connected, then all of them are connected.

Proof. (a) Suppose that $X$ is connected. Then, by $2.4 .3, \mathcal{F}_{n}(X)$ is connected 
for all $n$. If $E, F \in \mathcal{F}(X)$, then they both belong to some (connected!) $\mathcal{F}_{n}(X)$; hence $\mathcal{F}(X)$ is connected. But $\mathcal{f}(X) \subset \subseteq \subset \mathcal{A}(X)=\mathrm{Cl}(\mathcal{F}(X))$ (see 2.4.1), and therefore $\widetilde{S}$ is also connected.

(b) If $\mathcal{F}_{n}(X)$ or $\subseteq$ is connected, then, by $2.8, X$ is also connected.

Proposition 4.11. Let $A_{1}, \cdots, A_{n}$ be connected subsets of $X$. If $\mathcal{F}(X)$ $\cap\left\langle A_{1}, \cdots, A_{n}\right\rangle^{+} \subset \subseteq \subseteq\left\langle\left\langle\bar{A}_{1}, \cdots, \bar{A}_{n}\right\rangle^{+}\right.$, then $\subseteq$ is connected.

Proof. By Theorem $4.10, \mathcal{F}\left(A_{i}\right)$ is connected $(i=1, \cdots, n)$, and therefore $\mathcal{F}\left(A_{1}\right) \times \cdots \times \mathcal{F}\left(A_{n}\right)$ is connected in $[\mathcal{C A}(X)]^{n}$. But $\mathcal{F}(X) \cap\left\langle A_{1}, \cdots, A_{n}\right\rangle^{+}$is the image of $\mathcal{F}\left(A_{1}\right) \times \cdots \times \mathcal{F}\left(A_{n}\right)$ under the (continuous!) map discussed in 5.8.1, and therefore $\mathcal{f}(X) \cap\left\langle A_{1}, \cdots, A_{n}\right\rangle^{+}$is also connected. Finally $\subseteq$ is contained between a connected collection and its closure (see 2.3.2), and therefore $\subseteq$ is also connected. Q.E.D.

Theorem 4.12. Let $\mathcal{f}(X) \subset \subseteq \subset C(X)$. Then $X$ is locally connected if and only if $\mathfrak{S}$ is locally connected.

Proof. (a) Suppose that $X$ is locally connected. If $E \in \mathcal{C}(X)$, and if $\mathfrak{U}$ is a neighborhood of $E$ in $A(X)$, then we can find connected, open sets $U_{1}, \cdots, U_{n}$ such that $E \in\left\langle U_{1}, \cdots, U_{n}\right\rangle \subset \mathfrak{U}$. It now follows easily from Proposition 4.11 that $\subseteq$ is also locally connected.

(b) Suppose, conversely, that $\subseteq$ is locally connected. Let $x \in X$, and let $U$ be a neighborhood of $x$. Then there exists a connected neighborhood $\mathfrak{B}$ of $\{x\}$ in $\mathfrak{S}$, such that $\mathfrak{B C}\langle U\rangle$. Therefore $V=U_{A \in \mathfrak{B}} A$ is a neighborhood of $x$, $V \subset U$ and $\mathfrak{B}$ is connected (by 2.8) since $\{x\} \in \mathfrak{B}$ and $\{x\}$ is connected. Q.E.D.

Theorem 4.10 is false with the uniform topology on $A(X)$. (If $X$ is an unbounded metric space, with uniform structure $U$ induced by the metric, then the collection of bounded sets is open and closed in $\left(\mathcal{A}(X),\left|2^{U}\right|\right)$.) Theorem 4.12 becomes false if $\mathcal{C}(X)$ is replaced by $2^{X}$ or $\mathcal{A}(X)$. (If $X$ is the real line, $I$ the set of integers, and $\mathfrak{U}=\left\langle\bigcup_{n \in \mathrm{I}} S_{1 / 10}(n)\right\rangle$, then there is no connected neighborhood of $A$ contained in $\mathfrak{u}$.)

In the following proposition we collect some of the remaining facts about connectedness in $2^{x}$ and $\mathcal{C}(X)$. They are all easily verified. (We call a space totally disconnected if any two distinct points can be separated by an open and closed set.)

Proposition 4.13(7).

4.13.1. $X$ is zero-dimensional $\rightleftarrows \mathcal{C}(X)$ is zero-dimensional.

4.13.2. $X$ is totally disconnected $\rightleftarrows \mathcal{C}(X)$ is totally disconnected.

4.13.3. $X$ is discrete $\rightleftarrows \mathcal{C}(X)$ is discrete.

4.13.4. $X$ has no isolated points $\rightleftarrows 2^{X}$ has no isolated points.

4.13.5. The collection of connected elements of $2^{X}$ is closed in $2^{x}$.

5. Functions from or to hyperspaces. In this section, it will be useful to 
extend our topologies and uniform structures to $A(X)$ (see Notation 1.4). We make the following definition.

DeFinition 5.1.

5.1.1. If $\left\{U_{\imath}\right\}_{\iota} \in \mathrm{I}$ is a collection of subsets of a topological space $X$, then $\left\langle U_{\iota}\right\rangle_{\iota \in \mathrm{I}}^{+}$denotes $\left\{E \subset \mathcal{C}(X) \mid E \subset U_{\iota \in \mathrm{I}} U_{\imath} ; E \cap U_{\iota} \neq \varnothing \varnothing\right.$ for all $\left.\iota \in \mathrm{I}\right\}$. For a uniform space, $\mathfrak{B}_{\alpha}^{+}(E)$ denotes $\left\langle V_{\alpha}(x)\right\rangle_{x \in E}^{+}$.

5.1.2. The finite and uniform topologies on $\mathcal{A}(X)$ are defined just as in Definitions 1.6 and 1.7, except that $\langle-\rangle$ is now replaced by $\langle-\rangle^{+}$.

5.1.3. The map $\phi: \mathcal{A}(X) \rightarrow 2^{X}$ is defined by $\phi(A)=\bar{A}$.

In the following corollary, $T \mid A$ stands for the relative topology induced on $A$ by the topology $T$ on $X \supset A$. Similarly for uniform structures.

Corollary 5.2. Let $X$ be a topological (uniform) space. Then:

5.2.1. $\left\langle U_{\iota}\right\rangle_{\iota \in \mathrm{I}}=2^{x} \cap\left\langle U_{\imath}\right\rangle_{i \in \mathrm{I}}^{+}$.

5.2.2. The finite (uniform) topology on $2^{X}$ is the relative topology induced on $2^{x}$ by the finite (uniform) topology on $A(X)$.

5.2.3. If $(X, T)$ is a topological space and if $A \subset X$, then $\left(\mathcal{C}(A), 2^{T \mid A}\right)$ $=\left(\mathcal{A}(A), 2^{T} \mid 2^{A}\right)$.

5.2.3'. If $[X, U]$ is an uniform space, and if $A \subset X$, then $\left[\mathcal{C}(A), 2^{U \mid A}\right]$ $=\left[\mathcal{A} A(A), 2^{U} \mid 2^{A}\right]$.

5.2.4. If $A \subset X$ is dense, then $2^{A}$ is dense in $A(X)$ in the finite or uniform topology.

A fundamental relation between $A(X)$ and $2^{X}$ is established by the following theorem.

THEOREM 5.3.

5.3.1. If $(X, T)$ is normal, then $\phi$ is a retraction.

5.3.1'. If $[X, U]$ is uniform, then $\phi$ is a uniform retraction.

Proof. In both cases, $2^{x}$ evidently remains pointwise fixed. It remains to show the (uniform) continuity of $\phi$.

5.3.1. Let $E \in \mathcal{C}(X)$, and let $\mathfrak{U}=\left\langle U_{1}, \cdots, U_{n}\right\rangle$ be a neighborhood of $\bar{E}$. Pick $V_{0}$ such that $\bar{E} \subset V_{0}$ and $\bar{V}_{0} \subset \bigcup_{i=1}^{n} U_{i}$, and let $\mathfrak{B}=\left\langle V_{0} \cap U_{1}, \cdots\right.$, $\left.V_{0} \cap U_{n}\right\rangle^{+}$. Then $\mathfrak{B}$ is a neighborhood of $E$ in $\mathcal{A}(X)$, and $\phi(\mathfrak{B}) \subset \mathfrak{U}$.

5.3.1'. To index $\alpha$ for $2^{X}$, assign any $\beta \in \Sigma(\alpha)$ for $\mathcal{A}(X)$. Q.E.D.

Corollary 5.4. If $(X, T)$ is a topological space ( $[X, U]$ a uniform space), and $A \subset X$, then $\phi \mid 2^{A}$ is $1-1$ and continuous (uniformly continuous) with the finite (uniform) topology on both $2^{A}$ and $2^{X}$.

We now define some important functions and derive their properties.

DEFINITION 5.5.

5.5.1. $\sigma: \mathcal{C} A(\mathcal{C}(X)) \rightarrow \mathcal{A}(X)$ is defined by $\sigma(\mathfrak{B})=\mathrm{U}_{E \in \mathfrak{B}} E$.

5.5.2. $\theta: 2^{X} \rightarrow \mathcal{A}\left(2^{X}\right)$ is defined by $\theta(A)=\left\{E \in 2^{X} \mid E \subset A\right\}=2^{A}$.

5.5.3. $\eta: 2^{X} \rightarrow \mathcal{A}\left(2^{x}\right)$ is defined by $\eta(A)=\left\{E \in 2^{x} \mid E \cap A \neq \varnothing\right\}$. 
All statements in Corollary 5.6 are immediate consequences of previous results; following each statement, we list the relevant references.

COROllary 5.6.

5.6.1. If $X$ is regular, then $\sigma\left(\mathcal{C}\left(2^{X}, 2^{T}\right)\right) \subset 2^{X}$

5.6.2. $\sigma\left(\mathcal{C}\left(\mathcal{C}(X), 2^{T}\right)\right) \subset \mathcal{C}(X)$

5.6.3. $\theta\left(2^{X}\right) \subset 2^{\left(2^{X}, 2^{T}\right)}$

5.6.4. $\theta(\mathcal{C}(X)) \subset \mathcal{C}\left(\mathcal{C}(X), 2^{T}\right)$

5.6.5. $\eta\left(2^{X}\right) \subset 2^{\left(2^{X}, 2^{T}\right)}$

If $[X, U]$ is a uniform space, then

5.6.1'. $\sigma\left(\mathcal{C}\left(2^{x},\left|2^{U}\right|\right)\right) \subset 2^{x}$

5.6.2'. $\sigma\left(\mathcal{C}\left(\mathcal{C}(X),\left|2^{U}\right|\right)\right) \subset \mathcal{C}(X)$

5.6.3. $\theta\left(2^{X}\right) \subset 2^{\left(2^{X}, 2^{U} \mid\right)}$

5.6.4'. $\theta(\mathcal{C}(X)) \subset \mathcal{C}\left(\mathcal{C}(X),\left|2^{U}\right|\right)$

(5.6.4 and 3.3),

5.6.5'. $\eta(\mathcal{C}(X)) \subset 2^{\left(2^{X},\left|2^{I I}\right|\right)}$

$\left(2.2 .2^{\prime}\right)$.

THEOREM 5.7. If $[X, U]$ is a uniform space, and if all hyperspaces appearing in Definition 5.5 carry the uniform topology, then

5.7.1. The functions $\sigma, \theta$, and $\eta$ are all uniformly continuous.

If $(X, T)$ is a topological space, and if all hyperspaces in Definition 5.5 carry the finite topology, then:

5.7.2. $\sigma$ is continuous,

5.7.3. $\theta \mid \mathcal{C}(X)$ is continuous,

5.7.4. $\eta$ is continuous if $X$ is compact.

Proof. 5.7.1. The essential fact in the proofs of this statement is that the index sets for $X, \mathcal{A}(X)$, and $\mathcal{A}(\mathcal{A}(X))$ are all the same. For each function, we shall give a rule which assigns to each index for the range an index for the domain. The verification of this rule is straightforward for $\sigma$, and a little more complicated for $\theta$ and $\eta$.

$\sigma$ : To $\alpha$ for $A(X)$ assign $\alpha$ for $A(\mathcal{A}(X))$. Let $\mathfrak{B} \in \mathcal{A}(\mathcal{A}(X)), \sigma(\mathfrak{B})=B$. Let $\mathfrak{D} \in\left\langle V_{\alpha}(E)\right\rangle_{E \in \mathfrak{B}}^{+}, \sigma(\mathfrak{D})=D$. We must show that $D \in \mathfrak{B}_{\alpha}^{+}(B)$.

(a) $D \subset V_{\alpha}(B)$ : Let $d \in D$. Then there exists an $F \in \mathfrak{D}$ such that $d \in F$. But there exists an $E \in \mathfrak{B}$ such that $F \in \mathfrak{B}_{\alpha}^{+}(E)$. Therefore there exists an $e \in E \subset B$ such that $d \in V_{\alpha}(e)$.

(b) $D \cap V_{\alpha}(x) \neq \varnothing$ for all $x \in B$ : The proof is similar to the one for (a), and we omit it.

$\theta$ : To $\alpha$ for $2^{2^{X}}$ assign a $\beta \in \Sigma(\alpha)$ for $2^{x}$. Let $A \in 2^{x}, \theta(A)=\mathfrak{X}$. Let $\mathfrak{B} \in \mathfrak{B}_{\beta}(A)$, $\theta(B)=\mathfrak{B}$. We must show that $\mathfrak{B} \in\left\langle V_{\alpha}(E)\right\rangle_{E \in \mathfrak{R}}$ :

(a) $\mathfrak{B} \cap \mathfrak{B}_{\beta}(E) \neq \varnothing$ for all $E \in \mathfrak{A}$ : Let $E \in \mathfrak{A}$. Then $E \subset A$. Let $H=\{y \in B \mid y$ $\in V_{\beta}(x)$ for some $\left.x \in E\right\}$. Then $H \subset B$, whence $\bar{H} \subset \bar{B}=B$, and therefore $\bar{H} \in \mathfrak{B}$. We shall show that $\bar{H} \in \mathfrak{B}_{\alpha}(E)$ :

(1) $\bar{H} \cap V_{\alpha}(x) \neq \varnothing$ for all $x \in E$ : Let $x \in E$. Then there exists a $y \in H \subset \bar{H}$ such that $y \in V_{\beta}(x) \subset V_{\alpha}(x)$.

(2) $\bar{H} \subset V_{\alpha}(E)$ : Let $z \in \bar{H}$. Pick $y \in H$ such that $z \in V_{\gamma}(y)$, where $\gamma \in \Sigma(\beta)$. 
Next, pick $x \in E$ such that $y \in V_{\beta}(x)$. Then $z \in V_{\alpha}(x)$.

(b) $\mathfrak{B} \subset U_{E \in \mathfrak{T}} \mathfrak{B}_{\alpha}(E)$ : The proof is similar to that for (a), and we omit it. $\eta$ : To $\alpha$ for $2^{2^{X}}$ assign $\beta \in \Sigma(\alpha)$ for $2^{X}$ : The proof is similar to the one for $\theta$, and will be omitted.

5.7.2. Let $\mathfrak{B} \in \mathcal{A}(\mathcal{A}(X)), \sigma(\mathfrak{B})=B$, and let $\mathfrak{U}=\left\langle U_{1}, \cdots, U_{n}\right\rangle^{+}$be a neighborhood of $B$. We must find a neighborhood $\mathfrak{A}$ of $\mathfrak{B}$ such that $\sigma(\mathfrak{A}) \subset \mathfrak{U}$. Let $U=\bigcup_{i=1}^{n} U_{i}$, and let $\mathfrak{U}_{0}=\left\{E \in 2^{x} \mid E \subset U\right\}$; then $\mathfrak{U}_{0}$ is open in $2^{x}$, by 2.2.2. Let $\mathfrak{U}_{i}=\left\langle U_{i}, U\right\rangle^{+}(i=1, \cdots, n)$. We now define $\mathfrak{A}=\left\langle\mathfrak{U}_{0}, \mathfrak{u}_{1}, \cdots, \mathfrak{u}_{n}\right\rangle^{+}$.

(a) $\mathfrak{A}$ is a neighborhood of $\mathfrak{B}$ : Let $x_{i} \in U_{\boldsymbol{i}} \cap B$, and pick $B_{i} \in \mathfrak{B}$ such that $x_{i} \in B_{i}(i=1, \cdots, n)$. Then $B_{i} \in\left\langle U_{i}, X\right\rangle^{+}(i=1, \cdots, n)$, and $\mathfrak{B} \subset \mathfrak{U}_{0}$.

(b) $\sigma(\mathfrak{A}) \subset \mathfrak{U}$ : Let $\mathfrak{D} \in \mathfrak{A}$, and let $\sigma(\mathfrak{D})=D$.

(1) $D \subset U$ : Let $x \in D$. Then there exists an $E \in \mathfrak{D}$ such that $x \in E$. But $E \in \mathfrak{U}$, so $E \subset U$, whence $x \in U$.

(2) $D \cap U_{i} \neq \varnothing$ for all $i$ : Pick $E_{i} \subset \mathfrak{D}$ such that $E \in \mathfrak{U}_{i}(i=1, \cdots, n)$. Now pick $y_{i} \in E_{i}$ such that $y_{i} \in U_{i}(i=1, \cdots, n)$. Then $y_{i} \in D \cap U_{i}$ $(i=1, \cdots, n)$.

5.7.3. If $X$ is completely regular, then it admits a uniform structure, and our assertion follows from 5.7.1, 5.6.4, and 3.3. The assertion can be proved directly, without assuming complete regularity (but using the restriction to $\mathcal{C}(X)$ ). The proof is somewhat messy, and is omitted.

5.7.4. If $X$ is Hausdorff, then, as in 5.7.3, the assertion follows from 5.7.1, 4.2, and 3.3. Here again the assertion could be proved directly, without assuming Hausdorffness (but using the compactness of $X$ ). Q.E.D.

Concerning the function $\sigma$, we establish the following additional conclusions:

Corollary 5.8. Let $X$ be a topological (uniform) space. Then

5.8.1. The map from $[\mathcal{A}(X)]^{n}$ into $\mathcal{A}(X)$ which sends $\left(E_{1}, \cdots, E_{n}\right)$ into $\bigcup_{i=1}^{n} E_{i}$ is continuous (uniformly continuous) with the finite (uniform) topology on $A(X)$.

5.8.2. If, in 5.8.1, we map $(E, F)$ into $E \cap F$, the function is not continuous (uniformly continuous) (it is, however, upper semi-continuous; see Appendix). The same applies to the function which we get if $\cap$ replaces $\cup$ in the definition of $\sigma$.

\subsubsection{In either topology, $\sigma(\overline{\mathfrak{B}}) \subset \mathrm{Cl}(\sigma(\mathfrak{B}))$ for any $\mathfrak{B} \in \mathcal{A}(\mathcal{A}(X))$.}

Proof. 5.8.1. This follows from 5.7.2 (5.7.1) and 2.4.3. It is also easy to prove directly. Q.E.D.

We now study functions between topological spaces.

Definition 5.9. Let $f: X \rightarrow Y$ be onto. We define

5.9.1. $f^{*}: \mathcal{C}(X) \rightarrow \mathcal{A}(Y)$ by $f^{*}(E)=f(E)$.

5.9.2. $f^{-1 *}: Y \rightarrow \mathcal{A}(X)$ by $f^{-1 *}(y)=f^{-1}(y)$.

5.9.3. $f^{-1 * *}: \mathcal{C} A(Y) \rightarrow \mathcal{A}(X)$ by $f^{-1 * *}(E)=f^{-1}(E)$.

5.9.4. $\mathcal{D}(f)=\left\{f^{-1}(y)\right\}_{y \in Y}=f^{-1 *}(Y)$. 
Before stating the fundamental theorem on the functions defined above, we recall (make?) the following definition about uniform spaces which will be used in 5.10.2' below:

Let $f: X \rightarrow Y$ be a function between uniform spaces. Then $f$ is uniformly open (resp. closed) if to every index $\alpha$ for $X$ there corresponds an index $\alpha^{\prime}$ for $Y$, such that for every $x \in X \quad(y \in Y)$ we have $f\left(V_{\alpha}(x)\right) \supset V_{\alpha^{\prime}}(f(x))$ (resp. $\left.f\left[\left(V_{\alpha}\left(f^{-1}(y)\right)\right)^{\prime}\right] \subset\left(V_{\alpha^{\prime}}(y)\right)^{\prime}\right)$.

Theorem 5.10. Let $X, Y$ be topological spaces, and let $f: X \rightarrow Y$ be onto. Then' with the finite topology:

5.10.1. $f^{*}$ is continuous (a homeomorphism) if and only if $f$ is continuous (a homeomorphism).

5.10.2 $\left.{ }^{8}\right) . f^{-1 *}$ is continuous if and only if $f$ is open and closed.

5.10.3. $f^{-1 * *}$ is continuous if and only if $f^{-1 *}$ is continuous.

Now let $X, Y$ be uniform spaces. Then:

$5.10 .1^{\prime} . f^{*}$ is uniformly continuous (an isomorphism) if and only if $f$ is uniformly continuous (an isomorphism).

$5.10 .2^{\prime} . f^{-1 *}$ is uniformly continuous if and only if $f$ is uniformly open and uniformly closed.

5.10.3'. $f^{-1 * *}$ is uniformly continuous if and only if $f^{-1 *}$ is uniformly continuous.

If $\mathcal{D}(f)$ carries the factor topology, then

$5.10 .2^{\prime \prime}$. If $f$ is either open or closed, then $f^{-1 *}$ is continuous.

Finally we have

5.10.4. If $f$ is continuous, open, and closed, then the finite and the factor topology on $\mathcal{D}(f)$ are equivalent.

Proof. Assertions 5.10.1 and 5.10.1' follow directly from the definitions. 5.10.2" is well known and trivial. The proof for $5.10 .2^{\prime}\left(5.10 .3^{\prime}\right)$ is the same as for 5.10.2 (5.10.3). Finally, 5.10.4 follows immediately from 5.10.2 and 2.7.

5.10.2. (a) Suppose $f$ is open and closed. Let $y \in Y$, and let $f^{-1 *}(y)=E$. Let $\mathfrak{U}=\left\langle U_{1}, \cdots, U_{n}\right\rangle^{+}$be a neighborhood of $E$, and let $U=\bigcup_{i=1}^{n} U_{i}$. Let $V_{1}=\bigcup_{i=1}^{n} f\left(U_{i}\right)$; since $f$ is open, $V_{1}$ is open. Let $V_{2}=\left(f\left(U^{\prime}\right)\right)^{\prime} ;$ then $y \in V_{2}$, and $V_{2}$ is open since $f$ is closed. Letting $V=V_{1} \cap V_{2}$, we see that $f^{-1 *}(V) \subset \mathfrak{U}$.

(b) Suppose that $f^{-1}$ is continuous. If $U \subset X$ is open, then $f(U)$ $=\left(f^{-1 *}\right)^{-1}\left(\langle X, U\rangle^{+}\right)$which is open; so $f$ is open. If $A \subset X$ is closed, then $f(A)$ $=\left(\left(f^{-1 *}\right)^{-1}\left(\left\langle A^{\prime}\right\rangle^{+}\right)\right)^{\prime}$ which is closed; so $f$ is closed.

5.10.3. (a) If $f^{-1 * *}$ is continuous, so is $f^{-1 *}=f^{-1 * *} \mid \mathcal{F}_{1}(Y)$.

(b) If $f^{-1 *}$ is continuous, so is $f^{-1 * *}=\sigma_{0}\left(f^{-1 *}\right)^{*}$. Q.E.D.

From Theorem 5.10 it follows that if $X$ and $Y$ are homeomorphic (uniformly isomorphic) so are $2^{X}$ and $2^{Y}$. The converse, however, is false; see [5].

In the special case where the domain $X$ is compact, we have:

Proposition 5.11. Let $f: X \rightarrow Y$ be a continuous map from a compact Hausdorff space $X$ onto a Hausdorff space $Y$. Then: 
5.11.1. If $\mathcal{D}(f)$ is closed in $2^{x}$, then the finite topology on $\mathcal{D}(f)$ is equivalent to the factor topology.

5.11.2. $f^{-1 *}$ is continuous if and only if $\mathcal{D}(f)$ is closed in $2^{x}$.

Proof. 5.11.1. By Proposition 2.7, we need only show that the finite topology is coarser than the factor topology. Suppose $\mathbb{E} \subset \mathcal{D}(f)$ is closed in $\mathcal{D}(f)$ in the finite topology. Then $(E$ is compact, whence $\sigma(E)$ is closed (see 5.6.1); that is, $\mathbb{E}$ is closed in the factor topology.

5.11.2. If $\mathcal{D}(f)$ is closed in $2^{x}$, then $f^{-1 *}$ is continuous by 5.11 .1 and 5.10.2'. If $f^{-1 *}$ is continuous, then $\mathcal{D}(f)=f^{-1 *}(f(X))$ is compact, and hence closed.

From 5.10.2 and 5.11.2 we conclude that:

Corollary 5.12. If $f: X \rightarrow Y$ is a continuous map from a compact Hausdorff space $X$ into a Hausdorff space $Y$, then $f$ is open if and only if $\mathcal{D}(f)$ is closed in $2^{x}$.

Before concluding this section, we shall define an interesting new concept and derive its principal properties.

Definition 5.13. Let $(X, T)$ be a topological space, and let $\mathfrak{B} \in \mathcal{C}\left(2^{\boldsymbol{x}}\right)$.

5.13.1. The saturate of $\mathfrak{B}$ (written sat ( $\mathfrak{B})$ ) is $\left\{A \in 2^{X} \mid A\right.$ is a union of elements of $\mathfrak{B}\}$.

5.13.2. $\mathfrak{B}$ is saturated if sat $(\mathfrak{B})=\mathfrak{B}$.

As immediate consequences of this definition we have the following corollary.

Corollary 5.14. If $\mathfrak{B} \in \mathcal{A}\left(2^{x}\right)$, then:

5.14.1. sat $(\mathfrak{B}) \in \mathcal{A}\left(2^{x}\right)$.

5.14.2. sat $(\operatorname{sat}(\mathfrak{B}))=\operatorname{sat}(\mathfrak{B})$.

5.14.3. $\sigma(\operatorname{sat}(\mathfrak{B}))=\sigma(\mathfrak{B})$.

5.14.4. If $\mathfrak{B}$ is saturated, and if $A \in 2^{x}$, then $\mathfrak{B} \cap \theta(A)$ and $\mathfrak{B} \cap \eta(A)$ are both saturated.

We further have the following lemma.

Lемма 5.15. If $\mathfrak{B} \in \mathcal{C}\left(2^{x}\right)$, then, in the finite topology:

5.15.1. $\left\{A \in 2^{X} \mid A\right.$ is a finite union of elements of $\left.\mathfrak{B}\right\}$ is dense in sat (B).

5.15.2. If $\mathfrak{B}$ is saturated, then $\mathrm{Cl}(\sigma(\mathfrak{B})) \in \overline{\mathfrak{B}}$ (closure of $\mathfrak{B}$ in $2^{X}$ ).

5.15.3. If $\mathfrak{B}$ is saturated, then $\mathrm{Cl}(\sigma(\mathfrak{B}))=\sigma(\overline{\mathfrak{B}})$.

Proof. 5.15.1. Let $E \in$ sat $(\mathfrak{B})$, and let $\mathfrak{U}=\left\langle U_{1}, \cdots, U_{n}\right\rangle$ be a neighborhood of $E$. Pick $x_{i} \in U_{i} \cap E$; then there exists an $E_{i} \in \mathfrak{B}$ such that $E_{i} \subset E$ and such that $x_{i} \in E_{i}(i=1, \cdots, n)$, by the definition of saturate. But now $\bigcup_{i=1}^{n} E_{i} \in \mathfrak{U}$, proving the assertion.

5.15.2. Let $\mathfrak{U}=\left\langle U_{1}, \cdots, U_{n}\right\rangle$ be a neighborhood of $\mathrm{Cl}(\sigma(\mathfrak{B}))$. Pick $x_{i} \in U_{i} \cap \sigma(\mathfrak{B})$; then there exists an $E_{i} \in \mathfrak{B}$ such that $x_{i} \in E_{i}(i=1, \cdots, n)$. But then $\bigcup_{i=1}^{n} E_{i} \in \mathfrak{B} \cap \mathfrak{u}$. 
5.15.3. This follows from 5.15 .2 and 5.8.3. Q.E.D.

From 5.15.3. we have the following corollary, which should be compared to 2.5.1 and 2.5.2 (or 5.6.1 and 5.6.2).

Corollary 5.16. Let $(X, T)$ be a topological space. If $\mathfrak{B} \in 2^{\left(2^{X}, 2^{T}\right)}$ is saturated, then $\sigma(B) \in 2^{X}$; that is, a closed saturated union of closed sets is closed.

It is not true, in general, that the saturate of a closed collection is closed. For compact collections, however, we have the following proposition.

Proposition 5.17. If $X$ is a regular (uniform) space, and if $A\left(2^{X}\right)$ carries the finite (uniform) topology, then:

5.17.1. If $\mathfrak{B} \in \mathcal{C}\left(2^{X}\right)$, then sat $(\mathfrak{B}) \in \mathcal{C}\left(2^{X}\right)$.

5.17.2. The function sat: $\mathcal{C}\left(2^{X}\right) \rightarrow \mathcal{C}\left(2^{x}\right)$ is continuous (uniformly continuous).

Proof. By 5.6.4 (5.6.4 $), \theta\left(\mathcal{C}\left(2^{x}\right)\right) \subset \mathcal{C}\left(\mathcal{C}\left(2^{x}\right)\right)$; by 5.7.2 and 5.10.1 (5.7.1 and 5.10.1'), the fact that a continuous image of a compact space is compact, and by 5.6.1 (5.6.1 $)$, we have $\sigma^{*}\left(\mathcal{C}\left(\mathcal{C}\left(2^{x}\right)\right)\right) \subset \mathcal{C}\left(2^{x}\right)$. Furthermore, $\theta$ is continuous (uniformly continuous) by 5.7.3 (5.7.1), and $\sigma^{*}$ is continuous (uniformly continuous) by 5.7.2 and 5.10.1 (5.7.1 and 5.10.1'). Hence the composite function $\sigma^{*} \bigcirc \theta$ sends $\mathcal{C}\left(2^{x}\right)$ into $\mathcal{C}\left(2^{x}\right)$ and is continuous (uniformly continuous). To complete the proof, we shall show that if $\mathfrak{B} \in \mathcal{C}\left(2^{x}\right)$, then sat $(\mathfrak{B})=\sigma^{*} \theta(\mathfrak{B})$. Now $\sigma^{*} \theta(\mathfrak{B})=\left\{E \in 2^{X} \mid E\right.$ is the union of a compact subcollection of $\mathfrak{B}\}$; since each element of $\sigma^{*} \theta(\mathfrak{B})$ is closed (see above), we have

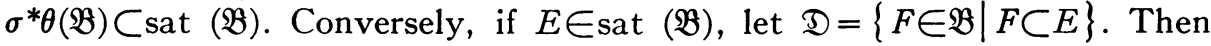
$E=\sigma(\mathfrak{D})$ and $\mathfrak{D} \in \theta(\mathfrak{B})$; hence $E \in \sigma^{*} \theta(\mathfrak{B})$, and hence sat $(\mathfrak{B}) \subset \sigma^{*} \theta(\mathfrak{B})$. Q.E.D.

This completes our study of the saturate. We conclude this section by stating, for later reference, the following lemma about general topology.

LEMMA 5.18. If $f: X \rightarrow Y$ is a closed function from the regular (topological) space $X$ onto the topological space $Y$, and if $f^{-1}(y)$ is closed (compact) for all $y \in Y$, then $f^{-1}(C)$ is closed (compact) for all compact $C \subset Y$.

Note $\left.{ }^{8}\right)$. If we assume that $f$ is open as well as closed, then the lemma follows from 5.10.2 and 2.5.1 (2.5.2).

6. Multi-valued functions. Suppose that $Y$ and $X$ are topological spaces, and that $g$ is a multi-valued function from $Y$ into $X$. It is then possible to regard $g$ as a single-valued function from $Y$ into $A(X)$, which is what we shall do from now on. Once $A(X)$ has been topologized, $g$ becomes a function between topological spaces, and it then makes sense to talk about its continuity and other topological properties. In this section we shall suppose that $A(X)$ carries the finite topology, and that, furthermore, $g$ maps $Y$ into $2^{X}$ (that is, that $g$, considered as a multi-valued function, maps each point of $Y$ into a closed subset of $X$ ).

We now ask the following question. 
Question 6.1. When is it possible to find a continuous $g^{\prime}: Y \rightarrow X$, such that $g^{\prime}(y) \in g(y)$ for all $y \in Y$ ?

A sufficient condition that this be possible is that

6.2. Both the following hold:

6.2.1. $g$ is continuous.

6.2.2. There exists a selection (see Definition 1.8) from $2^{x}$ to $X$.

The problem is thus reduced to two simpler ones; the second of which is concerned only with the space $X$, and has nothing to do with the space $Y$ or the function $g$. Most of $\$ 7$ will be devoted to determining what spaces satisfy 6.2.2.

Now suppose that $g$ has the special form $g=f^{-1 *}$, where $f: X \rightarrow Y$ is continuous. In this case, we call $g^{\prime}$ a continuous inverse for $f$, and we can improve the criteria of 6.2 as follows:

$6.2^{\prime}$. Both the following hold:

6.2'.1. $f$ is open and closed (see 6.1.1 and 5.10.2).

$6.2^{\prime} .2$. For an arbitrary disjoint covering $\mathfrak{D}$ of $X$ by closed sets, there exists a selection from $\mathfrak{D}$ to $X$.

In $\S 8$ we shall give a few preliminary results about spaces which satisfy $6.2^{\prime} .2$. We shall see there that there are spaces which satisfy $6.2^{\prime} .2$ but not 6.2.2.

Finally, suppose that, in addition, $X$ is compact Hausdorff. If now $f$ is a continuous function from $X$ onto a Hausdorff space $Y$, then any continuous inverse $g^{\prime}$ of $f$ is a homeomorphism of $Y$ into $X$, and $g^{\prime} \bigcirc f$ is a retraction of $X$ onto a subset homeomorphic to $Y$. We also have the following necessary and sufficient condition.

Proposition 6.3. Let $X$ be a compact Hausdorff space. Then the following are equivalent:

6.3.1. Every continuous open function from $X$ to a Hausdorff space $Y$ has a continuous inverse.

6.3.2. For every closed disjoint covering $\mathfrak{D}$ of $X$ by closed sets, there exists a selection from $\mathfrak{D}$ to $X$.

Proof. 6.3.1 $\rightarrow$ 6.3.2. Let $\mathfrak{D}$ be a closed disjoint covering of $X$ by closed sets. Let $i: X \rightarrow \mathfrak{D}$ be the cononical inclusion map; by 5.11 .1 it is continuous, and by 5.12 it is open. Now let $g^{\prime}$ be a continuous inverse for $i$; then $g^{\prime}$ is a selection from $\mathfrak{D}$ to $X$.

6.3.2 $\rightarrow$ 6.3.1. Let $f: X \rightarrow Y$ be continuous and open. Then $\mathscr{D}(f)$ is closed, by 5.12 , and $f^{-1 *}$ is continuous, by 5.10 .2 (or 5.11.2). Let $h: \mathcal{D}(f) \rightarrow X$ be a selection. Then $h \circ f^{-1 *}$ is a continuous inverse of $f$. Q.E.D.

7. Selection on $2^{X}$. Throughout this section, we assume that $(X, T)$ is a Hausdorff space, and that $2^{x}$ carries the finite topology.

We first prove some preliminary lemmas, from which our main results (in particular Theorem 1.9) will follow as corollaries. 
Notation. If $<$ is an order relation on $X$, then $I_{*}(x)=\{t \in X \mid t<x\}$; $I^{*}(x)=\{t \in X \mid t>x\}$.

Definition 7.1. If $f: \mathcal{F}_{2}(X) \rightarrow X$ is a selection, then $x<y$ means $x \neq y$ and $f(\{x, y\})=x\left({ }^{11}\right)$.

Our first lemma draws conclusions from the existence of a selection on $\mathcal{F}_{2}(X)$; it will soon be evident why $\mathcal{F}_{2}(X)$ plays a special part in our investigation.

Leмma 7.2. If $X$ is connected, and if there exists a selection $f: \mathcal{F}_{2}(X) \rightarrow X$, then:

7.2.1. For all $x \in X, I_{*}(x)$ and $I^{*}(x)$ are open in $X$.

7.2.2. < is a proper linear order on $X$.

7.2.3. The order topology on $X$ is coarser than $T$.

7.2.4. There exists exactly one other selection $g: \mathcal{F}_{2}(X) \rightarrow X$, namely

$$
g(\{x\})=x, g(\{x, y\})=\left\{\begin{array}{l}
x, \text { if } f(\{x, y\})=y, \\
y, \text { if } f(\{x, y\})=x .
\end{array}\right.
$$

Proof. 7.2.1. $I_{*}(x)$ : Let $t<x$. Then $f(\{t, x\})=t$. Let $U$ be a neighborhood of $t$ such that $x \in U$. Then, since $f$ is continuous, there exists a neighborhood $W_{1}$ of $t$ and a neighborhood $W_{2}$ of $x$ such that $f(F) \in U$ for all $F \in\left\langle W_{1}, W_{2}\right\rangle$. Hence $t^{\prime} \in W_{1} \rightarrow f\left(\left\{t^{\prime}, x\right\}\right) \in U \rightarrow f\left(\left\{t^{\prime}, x\right\}\right)=t^{\prime} \rightarrow t^{\prime}<x$.

$I^{*}(x)$. Let $t>x$. Then $f(\{t, x\})=x$. Let $U, V$ be open in $X$ such that $x \in U$, $t \in V, U \cap V=\varnothing$. By continuity of $f$, there exists a neighborhood $W_{1}$ of $x$, and a neighborhood $W_{2}$ of $y$ such that $F \in \mathfrak{W}=\left\langle W_{1}, W_{2}\right\rangle \rightarrow f(F) \in U$. Let $W=W_{2} \cap V$, and let $\mathfrak{W}^{\prime}=\left\langle W_{1}, W\right\rangle$. Then $\mathfrak{W}^{\prime} \subset \mathfrak{W}$, and therefore $F \in \mathfrak{W}^{\prime}$ $\rightarrow f(F) \in U$. Hence $t^{\prime} \in W \rightarrow f\left(\left\{x, t^{\prime}\right\}\right) \in U \rightarrow f\left(\left\{x, t^{\prime}\right\}\right)=x$ (since $t^{\prime} \in V$, so $\left.t^{\prime} \notin U\right) \rightarrow t^{\prime}>x$.

7.2.2. We need only show that $<$ is transitive. But this follows from 7.2.1 and $[6,(2.1)]$.

7.2.3. This follows immediately from 7.2.1.

7.2.4. (a) $g$ is a selection: If $x, y \in X$, write $x \ll y$ for $x \neq y, g(\{x, y\})=x$. Since $x \ll y \rightleftarrows y<x, 7.2 .2$ and 7.2.3 imply that $\ll$ is a proper linear order on $X$ such that the order $(\ll)$ topology is coarser than $T$. Applying Lemma 7.5.1, we find that $g$ is a selection.

(b) $f$ and $g$ are the only selections: Let $h$ be any selection on $\mathcal{F}_{2}(X)$. Then $h$ generates an order on $X$ such that the order topology is coarser than $T$ (by 7.2.2 and 7.2.3). We now apply [6, Theorem 2]; this tells us that the order generated by $h$ is the one generated by $f$ or its inverse. Hence $h=f$ or $h=g$. Q.E.D.

Lemma 7.3. Let $X$ be connected. Then:

7.3.1. If, for some fixed $n$, there exists a selection $f: \mathcal{F}_{n}(X) \rightarrow X$, then $f(E)$

(11) Strictly speaking, we should write $<_{f}$. Where no confusion can occur, we shall, however, omit this precaution. 
is the first $(<)$ element of $E$ for every $E \in \mathcal{F}_{n}(X)$.

7.3.2. If $\mathcal{F}(X) \subset \Im \subset 2^{x}$, and if there exists a selection $f: \Im \rightarrow X$, then $f(E)$ is the first $(<)$ element of $E$ for every $E \in \subseteq$.

Proof. 7.3.1. Let $B \in \mathcal{F}_{n}(X), x, y \in B$, and $x<y$. Let $\mathfrak{E}=\left\{E \in \mathcal{F}_{n}(X)\right.$ $\mid f(E \cup\{x, y\})=y\}$. We shall show that both $\&$ and $\mathcal{F}_{n}(X)-\mathbb{E}$ are open. Since $\mathcal{F}_{n}(X)$ is connected (by Theorem 4.10), and since $\{x, y\} \in \mathcal{F}_{n}(X)-\mathbb{E}$, it will follow that $₹$ is empty, and hence that $f(B) \neq y$. But this is the assertion of 7.3.1.

(a) $\mathcal{F}_{n}(X)-\mathfrak{E}$ open: Let $E \in \mathcal{F}_{n}(X)-($. Then $f(E \cup\{x, y\})=z \neq y$. Let $U$ be a neighborhood of $z$ such that $y \in U$. Then, since $f$ is continuous, there exists a neighborhood $\mathfrak{N}=\left\langle N_{1}, \cdots, N_{k}\right\rangle$ of $E \cup\{x, y\}$ such that $F \in \mathcal{F}_{n}(X)$ $\cap \mathfrak{l} \rightarrow f(F) \in U$. Let $\mathfrak{M}$ be a neighborhood of $E$ generated by those $N_{i}$ which are neighborhoods of some element of $E$. Since the only $N_{i}$ among the generators of $\mathfrak{N}$ which are not among the generators of $\mathfrak{M}$ are neighborhoods of $x$ or $y$, we have $F \in \mathcal{F}_{n}(X) \cap \mathfrak{M} \rightarrow F \cup\{x, y\} \in \mathfrak{N} \rightarrow f(F \cup\{x, y\}) \in U \rightarrow f(F \cup\{x, y\})$ $\neq y \rightarrow F \in \mathcal{F}_{n}(X)-\mathfrak{F}$.

(b) $\&$ is open: Let $E \in \mathbb{E}$. Then $f(E \cup\{x, y\})=y$. Let $U, V$ be open in $X$ such that $y \in U,[(E \cup\{x, y\})-\{y\}] \subset V$, and such that $U \cap V=\varnothing$. Then, by continuity of $f$, there exists a neighborhood $\mathfrak{N}=\left\langle N_{1}, \cdots, N_{k}\right\rangle$ of $E$ $\cup\{x, y\}$ such that $F \in \mathfrak{N} \cap \mathcal{F}_{n}(X) \rightarrow f(F) \in U$. Let $\mathfrak{M}=\left\langle M_{1}, \cdots, M_{j}\right\rangle$ be the neighborhood of $E$ which is derived from $\mathfrak{N}$ as in (a) above, and let $\mathfrak{M}^{\prime}=\left\langle M_{1} \cap V, \cdots, M_{j} \cap V\right\rangle$. Then $F \in \mathcal{F}_{n}(X) \cap \mathfrak{M}^{\prime} \rightarrow F \cup\{x, y\} \in \mathfrak{N}$ $\rightarrow f(F \cup\{x, y\}) \in U \rightarrow f(F \cup\{x, y\})=y$ (since the only point of $F \cup\{x, y\}$, which lies in $U$, is $y) \rightarrow F \in \mathbb{E}$.

7.3.2. Let $E \in \widetilde{S}, f(E)=y$. Suppose that the assertion of 7.3 .2 is false; then there exists an $x \in E$ such that $x<y$. Then $I^{*}(x)$ is a neighborhood of $y$, so there exists a neighborhood $\mathfrak{N}$ of $E$ such that $F \in \Im \cap \Re \rightarrow f(F) \in I^{*}(x)$. Now let $F$ be a finite set in $\mathfrak{S} \cap \mathfrak{N}$ (such an $F$ exists by Proposition 2.4.1) such that $x \in F$. Then $f(F)=z \in I^{*}(x), z \neq x$, so $x<z$. Let $F \in \mathcal{F}_{n}(X)$; then $f \mid \mathcal{F}_{n}(X)$ is a selection, and hence, by $7.3 .1, x>z$. We therefore have a contradiction. Q.E.D.

The assertions of the next lemma follow directly from Lemmas 7.3 and 7.2 , if we assume that $X$ is connected. To prove it under our weaker assumption, we use the fact that any set can be well-ordered.

LеммA 7.4. Suppose that all the connected components of $X$ are open. Then:

7.4.1. If there exists a selection $f: \mathcal{F}_{2}(X) \rightarrow X$, then there exists a linear order on $X$ such that the order topology is coarser than $T$.

7.4.2. If there exists a selection $f: 2^{X} \rightarrow X$, then there exists a linear order on $X$ such that the order topology is coarser than $T$, and such that every closed $(T)$ set has a first element.

Proof. Well order the components of $X$. For "the component which contains $x$ " we shall write " $C(x)$ ". 
7.4.1. It is evident that, for every component $C, f$ is a selection when restricted to $\mathcal{F}_{2}(C)=\left\{E \in \mathcal{F}_{2}(X) \mid E \subset C\right\}$. Define an ordering on $X$ as follows:

$x<y$ means $\left\{\begin{array}{l}f(\{x, y\})=x, \text { if } x \text { and } y \text { are in the same component, } \\ C(x)<C(y), \quad \text { if } x \text { and } y \text { are in different components. }\end{array}\right.$

This ordering is certainly linear, and furthermore, for every $x \in X$,

$$
\begin{aligned}
& I_{*}(x)=\{t \in C(x) \mid t<x\} \cap \underset{C<C(x)}{\cup} C, \text { which is open (see 7.2.1), } \\
& I^{*}(x)=\{t \in C(x) \mid t<x\} \cap \underset{C>C(x)}{\bigcup} C \text {, which is open (see 7.2.1). }
\end{aligned}
$$

7.4.2. Evidently, for every component $C, f$ is a selection on $2^{C}$ $=\left\{E \in 2^{X} \mid E \subset C\right\}$. We define our order as in 7.4.1; it remains to show that every closed $(T)$ set has a first element. Let $A$ be closed, and let $C$ be the first component of $X$ containing a point of $A$. Then $A \cap C$ is a nonempty closed subset of $C$, and therefore contains a first element $y$ by 7.3.2. But this $y$ is evidently the first element of $A$, which proves our assertion. Q.E.D.

Our final lemma shows that, under certain conditions, there actually exists a selection from a subcollection of $2^{x}$ to $X$. Its proof is quite independent of the previous lemmas of this section, which deal with the converse problem.

Lemma 7.5.1. Suppose that there exists a linear order on $X$ such that the order topology is coarser than $T$, and let

$\mathcal{H}(X)=\left\{E \in 2^{X} \mid\right.$ For every $F \in 2^{X}, E \cap F$ is empty or has a first element $\}$.

7.5.1. Then $f: \mathfrak{F}(X) \rightarrow X$, defined by $f(E)=$ first element of $E$, is a selection.

7.5.2. Then $\mathcal{C}(X, T) \subset \mathcal{H}(X)$.

Proof. 7.5.1. Let $E \in \mathfrak{F}(X)$, and let $f(E)=x$. Let $U$ be an open $(T)$ set containing $x$; we must find a neighborhood $\mathfrak{N}$ of $E$ such that $F \in \mathfrak{R} \cap \mathfrak{H C}(X)$ $\rightarrow f(F) \in U$. Now if $E \subset U$, then $\mathfrak{N}=\langle U\rangle$ is such a neighborhood, and we are through. Suppose, therefore, that $E \subset U$, and let $y$ be the first element of $E \cap U^{\prime}$. We now consider two cases:

(1) There is no $z \in X$ such that $x<z<y$. Let $N_{1}=\{t \mid t<y\} \cap U, N_{2}$ $=\{t \mid t>x\}$. Then $N_{1}$ and $N_{2}$ are open $(T) ; x \in N_{1}, y \in N_{2} ;$ and $E \subset N_{1} \cup N_{2}$. Hence $\mathfrak{N}=\left\langle N_{1}, N_{2}\right\rangle$ is a neighborhood of $E$. But $t_{1} \in N_{1}, t_{2} \in N_{2} \rightarrow t_{1}<z$, $t_{2} \geqq z \rightarrow t_{1}<t_{2} ;$ so $F \in \mathfrak{N} \cap \mathfrak{F C}(X) \rightarrow f(F) \in N_{1} \subset U$.

(2) There exists a $z \in X$ such that $x<z<y$ : Let $N_{1}=U, N_{2}=\{t \mid t<z\}$ $\cap U, N_{3}=\{t \mid t>z\}$. Then $N_{1}, N_{2}$, and $N_{3}$ are open $(T) ; x \in N_{1}, x \in N_{2}$, and $y \in N_{3}$. Also $E \in N_{1} \cup N_{2} \cup N_{3}$; for if $z \in E$, then $z \in U$ by definition of $y$ and $z$. Hence $\mathfrak{R}=\left\langle N_{1}, N_{2}, N_{3}\right\rangle$ is a neighborhood of $E$. Now $t_{2} \in N_{2}, t_{3} \in N_{3} \rightarrow t_{2}<t_{3}$; and therefore $F \in \mathfrak{R} \cap \mathfrak{F}(X) \rightarrow f(F) \in N_{1} \cup N_{2} \subset U$.

7.5.2. Let $E \in \mathcal{C}(X)$. Then $E \cap F$ is compact $(T)$ for all $F \in 2^{X}$, and hence is also compact in the order topology, whence it has a first point. Hence 
$\mathcal{C}(X)<\mathfrak{H C}(X)$. Q.E.D.

We are now ready to prove the principal results of this section. The proofs will consist of references to the lemmas from which they follow.

Proof of Theorem 1.9.

1.9.1 $\rightarrow$ 1.9.2: (7.4.2).

$1.9 .2 \rightarrow 1.9 .1:(7.5 .1)\left({ }^{12}\right)$.

$1.9 .3 \rightarrow$ 1.9.4: (7.4.1).

1.9.4 $\rightarrow$ 1.9.3: $\left(7.5 .1\right.$ and 7.5.2) $\left({ }^{12}\right)$ Q.E.D.

The next proposition deals with the possibility of extending a selection from $\mathcal{F}_{2}(X)$ to $X$.

Proposition 7.6. If $X$ is connected, and if there exists a selection $f: \mathscr{F}_{\mathbf{2}}(X)$ $\rightarrow X$, then:

7.6.1. There exists an extension of $f$ to $\mathcal{C}(X)$.

7.6.2. If every $E \in 2^{Y}$ has a first $\left(<_{f}\right)$ element, then there exists an extension of $f$ to $2^{x}$.

7.6.3. If $f(X) \subset \subseteq \subset 2^{X}$, then any extension to $\subseteq$ is unique.

7.6.4. For all $n$, any extension to $\mathcal{F}_{n}(X)$ is unique.

Proof. 7.6.1: (7.2.2, 7.2.3, 7.5.1, and 7.5.2).

7.6.2: (7.2.2, 7.2.3, and 7.5.1).

7.6.3: (7.3.2).

7.6.4: (7.3.1). Q.E.D.

The conclusions of Proposition 7.6 are easily combined to yield the following corollary.

CoROllary 7.7. Under the assumptions of Proposition 7.6, there exists a unique extension of $f$ to $\subseteq<2^{x}$, provided $\subseteq$ satisfies any one of the following:

7.7.1. $\subseteq=\mathcal{F}_{n}(X)$ for some $n$ (by 7.6.1 and 7.6.2).

7.7.2. $\mathcal{f}(X) \subset \subseteq \subset \mathcal{C}(X)$ (by 7.6.1 and 7.6.3).

7.7.3. $\mathcal{f}(X) \subset \subseteq$, and every $E \in 2^{X}$ has a first $\left(<_{f}\right)$ element (by 7.6.2 and 7.6.3).

The final proposition of this section gives the number of different selections that may exist from a subcollection of $2^{x}$ to $X$.

Proposition 7.8. Let $X$ be connected. Let $k$ stand for the maximal number of different selections that can exist from a fixed subcollection $\subseteq$ of $2^{x}$ to $X$. Then:

7.8.1. If $\mathfrak{F}_{2}(X) \subset \subseteq \subset \mathcal{C}(X)$, then $k=0$ or $k \geqq 2$.

7.8.2. If $\subseteq \supset \mathcal{F}(X)$, or if $\mathfrak{S}=\mathcal{F}_{n}(X)$ for some $n$, then $k \leqq 2$.

Proof. 7.8.1: (7.2.4, and 7.6.1).

7.8.2: (7.2.4, and 7.6.3 (or 7.6.4)). Q.E.D.

We conclude this section by giving some examples of spaces which do,

${ }^{(2)}$ A different criterion for the existence of a selection on $2^{x}$ or $\mathcal{C}(X)$ is given in Proposition 8.3. 
or do not, satisfy condition 1.9.2 and/or 1.9.4. We begin by observing that a linearly ordered space, which is connected in the order topology, becomes disconnected by the removal of any point except, possibly, the two end points. Hence $R^{n}$ (that is, Euclidean $n$-space), $\left\{x \in R^{n} \mid\|x\| \leqq 1\right\}$, and $\left\{x \in R^{n} \mid\|x\|=1\right\}$ do not satisfy either 1.9 .2 or 1.9 .4 for $n \geqq 2$. The real line $R^{1}$ satisfies 1.9 .4 ; it does not satisfy 1.9 .2 , as is easily seen by looking at a compact neighborhood of that point of $R^{1}$ which would have to become the first point in the ordering. Finally, a subset of $R^{1}$ which has either a first or a last element in the natural ordering satisfies both 1.9.2 and 1.9.4.

8. More on selections. We shall again assume that $(X, T)$ and $\left(Y, T^{\prime}\right)$ are Hausdorff spaces, and that $2^{X}$ and $2^{Y}$ carry the finite topology.

Definition 8.1. We shall call $X$ an $\left(S_{1} / S_{2} / S_{3} / S_{4}\right)$ space, if there exists a selection from $\left(2^{X} / \mathcal{C}(X) /\right.$ an arbitrary disjoint covering of $X$ by closed sets/an arbitrary disjoint covering of $X$ by compact sets) to $X$.

Lemma 8.2. Let $f: X \rightarrow Y$ be continuous, open, closed, and onto. Then, if

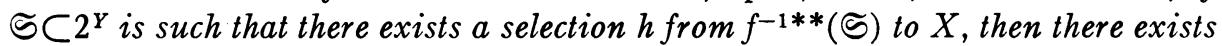
a selection from $\subseteq$ to $Y$.

Proof. The function $f \circ h \circ f^{-1 * *}$ is such a selection (see 5.10.2). Q.E.D.

Proposition 8.3. Let $f: X \rightarrow Y$ be continuous, open, closed, and onto. Then:

8.3.1. If $X$ is an $S_{i}$ space, then $Y$ is an $S_{i}$ space $(i=1,3)$.

8.3.2. If $X$ is an $S_{i}$ space, and if $f^{-1}(y)$ is compact for all $y \in Y$, then $Y$ is an $S_{i}$ space $(i=2,4)$.

Proof. This follows from Lemma 8.2 and, for 8.3.2, from Lemma 5.18. Q.E.D.

The remaining results of this section deal with $S_{3}$ and $S_{4}$ spaces. They are of a preliminary nature, and are stated without proof.

Lemma 8.4. If $X$ contains a subset which is homeomorphic to a circle, then $X$ is not an $S_{4}$ (and therefore not an $S_{3}$ ) space.

Definition 8.5.

8.5.1. A tree is a partially ordered set $X$, such that $\{t \mid t \leqq x\}$ is simply ordered for all $x \in X$, and such that any two elements of $X$ have a g.l.b.

8.5.2. A branch point of a tree $X$ is a point $x \in X$ which is the g.l.b. of two distinct points of $X$, both of which are different from $x$.

8.5.3. The partial order topology $P$ on a tree $X$ is generated by sub-basic open sets of the following two kinds:

$$
\begin{array}{ll}
\{y \mid y \quad<x\}, & x \in X, \\
\{y \mid y \text { is not } \leqq x\}, & x \in X .
\end{array}
$$

Lemma 8.6. Suppose that $(X, T)$ can be partially ordered as a tree, such that the order topology $P$ is coarser than $T$, and such that $X$ has only a finite number 
of branch points. Then:

8.6.1. $X$ is an $S_{4}$ space.

8.6.2. If, in addition, every closed $(T)$ linearly ordered subset of $X$ has a last element, then $X$ is an $S_{3}$ space.

We conclude by observing that a finite tree, in the sense of homology theory (for instance a closed figure $X$ in the plane), satisfies 8.6.1 and 8.6.2. Unless this tree has only two vertices, however, it will not satisfy conditions 1.9.2 or 1.9.4 of Theorem 1.9.

Appendix. We begin by re-examining the foundations of the finite topology.

Consider a topological space $(X, T)$. It is easily seen (see [7] and Definition 1.8a, §2) that the finite topology on $A(X)$ (see Definition 5.1) is the join (or sup), in the lattice of all topologies on $\mathcal{A}(X)$, of the following two topologies.

Definition 9.1. The upper (lower) semi-finite topology on $A(X)$ is generated by taking as a basis (resp. sub-basis) for the open collections in $A(X)$ all collections of the form $\{E \in \mathcal{C}(X) \mid E \subset U\}$ (resp. $\{E \in \mathcal{A}(X) \mid E \cap U \neq \varnothing\}$ ) with $U$ an open subset of $X$. (To restrict these topologies to $2^{x}$, we merely replace $A(X)$ by $2^{x}$ in the above.)

It is clear that these topologies (as well as their meet in the lattice of all topologies on $A(X)$, which we shall call the hemi-semi-finite topology) are in general not $T_{1}$, and that they are admissible with respect to the generating topology on $X$.

If $X$ and $Y$ are topological spaces, we call a function $f: X \rightarrow \mathcal{A}(Y)$ upper (lower) semi-continuous if $f$ is continuous with the upper (lower) semi-finite topology on $A(Y)$. This condition on $f$ is equivalent to upper (lower) continuity of $f$ in the sense of [9, pp. 148-149], and to the strong upper (lower) semi-continuity, in the sense of $[5, \mathrm{pp} .70-71]$, of the relation $R(X, Y)$ defined by $R(x, y) \rightleftarrows y \in f(x)$. The condition must be distinguished from another condition, which sometimes bears the same name, defined in terms of the lim sup (lim inf) of sets in $Y$ or of $f$ (see [13, p. 148], [9, pp. 104-105], [5, p. $60]$, and $[4, \S I]$ which gives the simplest definition). This latter condition cannot, in general, be interpreted as continuity with respect to some topology on $A(Y)$. The connection between the conditions is established in $[4, \S \mathrm{I}]$.

The following theorem follows from the definitions.

THEOREM 9.2. If $X$ and $Y$ are topological spaces, then a function $f: X$ $\rightarrow \mathcal{A}(Y)$ is upper (lower) semi-continuous if and only if $\{x \in X \mid f(x) \cap A \neq 0\}$ is closed (open) in $X$ whenever $A$ is closed (open) in $Y$.

It is clear that $f$ is continuous with the finite topology on $A(Y)$ if and only if $f$ is both upper and lower semi-continuous. We therefore have the following corollary. 
Corollary 9.3. If $X, Y$ are topological spaces, then a function $f: X \rightarrow \mathcal{A}(Y)$ is continuous with the finite topology on $\mathcal{A}(Y)$ if and only if $\{x \in X \mid f(x)$ $\cap A \neq \varnothing\}$ is closed in $X$ whenever $A$ is closed in $Y$, and is open in $X$ whenever $A$ is open in $Y$.

Theorem 9.2 and Corollary 9.3 give useful criteria for the continuity of a function into a hyperspace. Corollary 9.3 should have been stated at the beginning of $\$ 5$; several subsequent proofs could then have been simplified (5.10.2, for instance, follows directly from Corollary 9.3). The author, who was not aware of this when $\$ 5$ was written, apologizes to the reader for the inconvenience which this omission may have caused him.

For the special case where $f\left(x_{1}\right) \cap f\left(x_{2}\right)=\varnothing$ for $x_{1} \neq x_{2}$, Theorem 9.2 yields the following improvement on 5.10.2.

COROLlary 9.4. With the notation of 5.10.2, $f^{-1 *}$ is upper (lower) semicontinuous if and only if $f$ is closed (open).

We close this survey of semi-continuous functions with the following curious result which is essentially due to $[5$, p. 70].

Proposition 9.4. If $X$ is first countable at $x_{0}$, if $Y$ is completely regular, and if $f: X \rightarrow \mathcal{A}(Y)$ is upper semi-continuous at $x_{0}$, then there exists a compact subset $C$ of $f\left(x_{0}\right)$ with the following property: If $V$ is an open set containing $C$, then there exists a neighborhood $U$ of $x_{0}$ such that $f(x) \subset V \cup f\left(x_{0}\right)$ for any $x \in U$.

To prove this proposition, look carefully at the sets $A_{n}=\mathrm{Cl}\left(\mathrm{U}_{f}(x)\right) \cap f\left(x_{0}\right)$, where the union is taken over all $x \in S_{1 / n}\left(x_{0}\right)(n=1,2, \cdots)$.

Let us now see how some of the results in $\$ 2$ can be strengthened by means of our new topologies. Proposition 2.7, for instance, remains true if the finite topology is replaced by any of the above weaker ones. (Even the hemi-semi-finite topology may be strictly finer than the factor topology on D); see Example 2.10.) The same is true of Proposition 2.8, if we demand that every element of $\mathfrak{B}$ should be connected. The most useful new result, however, is the following theorem.

TheOREM 9.5. Assertions 2.5.1 and 2.5.2 of Theorem 2.5 remain true (with unchanged proofs) if the finite topology is replaced by the upper semi-finite topology.

Corollary 9.6. If $X$ is a compact space, $Y$ a regular (topological) space, if $f: X \rightarrow A(Y)$ is upper semi-continuous, and if $f(x)$ is closed (compact) in $Y$ for all $x \in X$, then $\cup_{x \in X} f(x)$ is closed (compact) in $Y$.

The last two results provide a systematic method for attacking various problems. We give two examples:

1. Lemma 5.18 follows immediately from Corollary 9.6 and one part of Corollary 9.4 (see the remark following the lemma). 
2. In [8], the proof of (3) in Theorem 1 is reduced to proving the following assertion: If $X$ is a compact Hausdorff space, $Y$ a topological space, $F$ a collection of continuous functions from $X$ into $Y$ which is compact in the compact-open topology, and if $A$ is closed in $Y$, then $U_{f \in F f^{-1}(A)}$ is closed in $X$. This assertion follows at once from Corollary 9.6, when we observe that the mapping $\phi: F \rightarrow 2^{X}$, defined by $\phi(f)=f^{-1}(A)$, is upper semi-continuous.

Finally, let us glance at the uniform case. If $[X, U]$ is a uniform space with index set $\mathrm{A}$, then $2^{U}$ on $A(X)$ is clearly the join, in the lattice of all uniform structures on $A(X)$, of the following two uniform structures.

Definition 9.7. The upper (lower) semi-uniform structure on $\mathcal{A}(X)$ is generated by the index set $\mathbf{A}$, and the neighborhoods $\mathfrak{u}_{\alpha}(E)=\left\{F \mid F \subset \cdot V_{\alpha}(E)\right\}$ (resp. $\left.\mathfrak{W}_{\alpha}(E)=\left\{F \mid E \subset V_{\alpha}(F)\right\}\right)$ for $E \in \mathcal{A}(X)$.

These uniform structures are clearly not $T_{1}$, in general, and they are admissible with respect to $U$. We call the corresponding topologies the upper (lower) semi-uniform topologies.

It is clear that the upper (lower) semi-uniform topology is coarser (finer) than the upper (lower) semi-finite topology. Furthermore, it follows from Lemma 3.2 that the respective topologies agree on $\mathcal{C}(X)$; this generalizes Theorem 3.3.

Much of what we have said for the finite case has its analogue in the uniform case. (Proposition 9.4 does not.) In particular, we have the following theorem.

THEQREM 9.8. Assertions 2.5.1' and 2.5.2' of Theorem 2.5 remain true if the uniform topology is replaced by the upper semi-uniform topology.

If $X$ is a uniform space, then Theorems 9.8 and 9.6 make equivalent assertions about collections of compact sets; for collections of closed sets, however, the assertion in Theorem 9.8 is the stronger one.

Finally, it is interesting to note that, in our applications, Theorem 2.5 itself was sufficient for the uniform topology, while for the finite topology we needed the improvement in Theorem 9.6.

\section{BIBLIOGRAPHY}

1. N. Bourbaki, Topologie generale, Paris, Hermann 1940, chaps. 1 and 2.

2. - Topologie generale, Paris, Hermann, 1942, chaps. 3 and 4.

3. - Topologie generale, Paris, Hermann 1948, chap. 9.

4. R. Brissac, Sur les fonctions multiformes, C. R. Acad. Sci. Paris vol. 224 (1947) pp. 92-94.

5. G. Choquet, Convergences, Annales de l'Université de Grenoble vol. 23 (1947-1948) pp. $55-112$.

6. S. Eilenberg, Ordered topological spaces, Amer. J. Math. vol. 63 (1941) pp. 39-45.

7. O. Frink, Topology in lattices, Trans. Amer. Math. Soc. vol. 51 (1942) pp. 569-582.

8. D. Gale, Compact sets of functions and function rings, Proceedings of the American Mathematical Society vol. 1 (1950) pp. 303-308.

9. H. Hahn, Reele Funktionen, Akademische Verlagsgesellschaft, Leipzig, 1932.

10. F. Hausdorff, Mengenlehre, 3d ed., Springer, Berlin, 1927. 
11. J. L. Kelley, Hyperspaces of a continuum, Trans. Amer. Math. Soc. vol. 52 (1942) pp. 23-36.

12. C. Kuratowski, Topologie I, Monografje Matematiczne, Warsaw, 1933.

13. - Les fonctions semi-continues dans l'espace des ensembles fermes, Fund. Math. vol. 18 (1932) pp. 148-160.

14. L. Vietoris, Bereiche Zweiter Ordnung, Monatshefte für Mathematik und Physık vol. 33 (1923) pp. 49-62.

15. - Kontinua zweiter Ordnung, Monatshefte für Mathematik und Physik vol. 33 (1923) pp. 49-62.

16. A. Weil, Sur les espace a structure uniforme et sur la topologie generale, Paris, Hermann, 1937.

University of Chicago,

Chicago, Ill. 